\title{
Deformation Quantization of Poisson Structures Associated to Lie Algebroids ${ }^{\star}$
}

\author{
Nikolai NEUMAIER and Stefan WALDMANN
}

Fakultät für Mathematik und Physik, Albert-Ludwigs-Universität Freiburg, Physikalisches Institut, Hermann Herder Straße 3, D-79104 Freiburg, Germany

E-mail: Nikolai.Neumaier@physik.uni-freiburg.de,Stefan.Waldmann@physik.uni-freiburg.de

URL: http://idefix.physik . uni-freiburg.de/ nine/, http://idefix.physik.uni-freiburg.de/ $\sim$ stefan/

Received September 26, 2008, in final form May 25, 2009; Published online July 16, 2009 doi:10.3842/SIGMA.2009.074

\begin{abstract}
In the present paper we explicitly construct deformation quantizations of certain Poisson structures on $E^{*}$, where $E \longrightarrow M$ is a Lie algebroid. Although the considered Poisson structures in general are far from being regular or even symplectic, our construction gets along without Kontsevich's formality theorem but is based on a generalized Fedosov construction. As the whole construction merely uses geometric structures of $E$ we also succeed in determining the dependence of the resulting star products on these data in finding appropriate equivalence transformations between them. Finally, the concreteness of the construction allows to obtain explicit formulas even for a wide class of derivations and selfequivalences of the products. Moreover, we can show that some of our products are in direct relation to the universal enveloping algebra associated to the Lie algebroid. Finally, we show that for a certain class of star products on $E^{*}$ the integration with respect to a density with vanishing modular vector field defines a trace functional.
\end{abstract}

Key words: deformation quantization; Fedosov construction; duals of Lie algebroids; trace functionals

2000 Mathematics Subject Classification: 53D55; 53D17

\section{Introduction}

The question of existence and classification of formal star products deforming an arbitrary Poisson bracket on a Poisson manifold has been answered positively with the aid of Kontsevich's famous formality theorem [30], see also [10] for a Fedosov-like globalization. Besides the regular or even symplectic case, which was very well understood long before, see [12, 17, 41], up to then the quantity of examples of truly Poisson structures, that were known to be deformation quantizable, was very poor: essentially the constant and the linear Poisson brackets on a vector space. The linear Poisson brackets are precisely the Kirillov-Kostant-Souriau brackets on the dual $\mathfrak{g}^{*}$ of a Lie algebra $\mathfrak{g}$. A star product for this Poisson bracket has been obtained in the early work of Gutt [22]. Even after the general existence results the number of examples that could be 'handled' concretely remained small whence there is a justified interest in such concrete examples.

In the present paper we consider Poisson brackets and their deformation quantizations that in some sense generalize both canonical Poisson brackets on cotangent bundles, see also [2, 4, 3], and linear Poisson brackets on $\mathfrak{g}^{*}$, see [22]. These extreme examples can be merged into the Poisson geometry of $\left(E^{*}, \theta_{E}\right)$, where $E \longrightarrow M$ is a Lie algebroid and $\theta_{E}$ is the corresponding Poisson tensor on the dual bundle. One main point of our construction, that consists in a modified

${ }^{\star}$ This paper is a contribution to the Special Issue on Deformation Quantization. The full collection is available at http://www.emis.de/journals/SIGMA/Deformation_Quantization.html 
Fedosov procedure, is that it is elementary in the sense that it only relies on tensor calculus and avoids the use of the formality theorem. In addition, there are even more Poisson brackets that depend on an additional $\mathrm{d}_{E}$-closed $E$-two-form accessible by our method than just the canonical one. Moreover, it is geometric and permits to investigate various properties of the constructed products.

Yet another independent motivation for this particular class of Poisson structures and corresponding star products is given as follows. Let $E^{*} \longrightarrow M$ be the dual of a vector bundle $E \longrightarrow M$ and let $\xi \in \Gamma^{\infty}\left(T E^{*}\right)$ be the Euler vector field on $E^{*}$.

Definition 1.1 (Homogeneous star product). A star product $\star$ on $E^{*}$ is called homogeneous if $\mathrm{H}=\mathscr{L}_{\xi}+\lambda \frac{\partial}{\partial \lambda}$ is a derivation of $\star$.

Such homogeneous star products played a crucial role in the first existence proofs on cotangent bundles, see $[7,13]$. The homogeneous star products enjoy very nice features which makes them an interesting class to study. The following properties are verified in complete analogy to the case of cotangent bundles:

Proposition 1.2. Let $\star=\sum_{r=0}^{\infty} \lambda^{r} C_{r}$ be a homogeneous star product on $E^{*}$.

i) The Poisson structure $\{f, g\}_{\star}=\frac{1}{\mathrm{i}}\left(C_{1}(f, g)-C_{1}(g, f)\right)$ is a linear Poisson structure whence $E$ is a Lie algebroid.

ii) Let $f, g \in \operatorname{Pol}^{\bullet}\left(E^{*}\right)$ be of degree $k$ and $\ell$, respectively. Then

$$
f \star g=\sum_{r=0}^{k+\ell} \lambda^{r} C_{r}(f, g) \quad \text { with } \quad C_{r}(f, g) \in \mathrm{Pol}^{k+\ell-r}\left(E^{*}\right) .
$$

iii) The polynomial functions $\operatorname{Pol}^{\bullet}\left(E^{*}\right)[\lambda]$ are a $\mathbb{C}[\lambda]$-algebra with respect to $\star$. In particular, * converges trivially on $\mathrm{Pol}^{\bullet}\left(E^{*}\right)[\lambda]$ for all $\lambda=\hbar \in \mathbb{C}$.

$i v)$ The polynomial functions $\mathrm{Pol}^{\bullet}\left(E^{*}\right)[\lambda]$ are generated by $\operatorname{Pol}^{0}\left(E^{*}\right)[\lambda]$ and $\operatorname{Pol}^{1}\left(E^{*}\right)[\lambda]$ with respect to $\star$.

v) $\operatorname{Pol}^{0}\left(E^{*}\right)$ is a subalgebra with $\star$ being the undeformed commutative product.

It was brought to our attention by Simone Gutt that the homogeneity arguments from $[7,13]$ should also apply to our more general situation beyond the cotangent bundle case yielding an existence proof independent of our approach.

Following a suggestion of the referee, one may also wonder whether on a particular geometry like a vector bundle $E^{*} \longrightarrow M$ there is a simplified construction of a formality which would allow for a construction of a star product for the linear Poisson structure analogously to the approach of Dito [14] or Kathotia [29]. We leave this question for a future investigation.

For the case of an integrable Lie algebroid there is yet another approach to quantization namely that of strict deformation quantization considered in [34] which is based on a generalized Weyl quantization using pseudo-differential operators. Also for the non-integrable case using the local integrating Lie groupoids, the latter product has been related to the universal enveloping algebra associated to the Lie algebroid in [40]. It yields a star product on the polynomial functions on $E^{*}$. Actually, it is not evident, see e.g. [3], but in fact true, that this product extends to a well-defined bidifferential formal star product on all smooth functions $C^{\infty}\left(E^{*}\right)$. Also Chemla discussed, in the framework of complex Lie algebroids, the relation between Lie algebroids and certain algebras of differential operators, see e.g. [11]. Finally, one should note that although there are some similarities our setting and our construction are different from that in [36], where symplectic Lie algebroids are quantized and not the dual $E^{*}$ with its in general truly non-symplectic Poisson structure. 
Our paper is organized as follows: in Section 2 we collect some preliminaries on Lie algebroids $E \longrightarrow M$ and associated Poisson brackets on $E^{*}$ to fix our notation. In Section 3 we present our construction of star products on the polynomial functions on $E^{*}$. We show that they actually quantize the given Poisson brackets and that they extend in a unique way to star products on all smooth functions. Our construction depends on an ordering parameter $\kappa$, an $E$-connection $\nabla$, and a formal series of $\mathrm{d}_{E}$-closed $E$-two-forms $B$. Section 4 is devoted to a further investigation of the products. In particular, we show that among our star products there are homogeneous star products. In Section 5 we explicitly construct equivalence transformations between the products provided the parameters are appropriately related. In Section 6 we make contact to the results of [40] and relate the products obtained in the particular case $B=0$ to the universal enveloping algebra of $E$. Finally, Section 7 shows that in the case of a unimodular Lie algebroid the classical trace consisting in the integration with respect to a certain constant density on $E^{*}$ is also a trace functional for any homogeneous star product on $E^{*}$. Here we establish a relation between the a priori different notions of unimodularity of the Lie algebroid $E$ and the Poisson manifold $\left(E^{*}, \theta_{E}\right)$ that is interesting for its own, see also [44, Section 7].

\section{Preliminaries on linear Poisson structures and Lie algebroids}

In this section we collect some well-known facts on linear Poisson structures and Lie algebroids in order to fix our notation. For details see e.g. [35, 33, 9].

Let $E \longrightarrow M$ be a vector bundle of fibre dimension $N$ over an $n$-dimensional manifold. Then $E$ is a Lie algebroid if it is equipped with a bundle map, the anchor $\varrho: E \longrightarrow T M$, and a Lie bracket $[\cdot, \cdot]_{E}$ for the sections $\Gamma^{\infty}(E)$ in $E$ such that we have the Leibniz rule $[s, u t]_{E}=$ $u[s, t]_{E}+(\varrho(s) u) t$ for all $u \in C^{\infty}(M)$ and $s, t \in \Gamma^{\infty}(E)$. It follows that $\varrho\left([s, t]_{E}\right)=[\varrho(s), \varrho(t)]$. There are (among many others) three examples of interest:

\section{Example 2.1 (Lie algebroids).}

i) Clearly $T M$ with $\varrho=$ id and $[\cdot, \cdot]_{T M}$ the canonical Lie bracket is a Lie algebroid. Thus Lie algebroids over $M$ generalize the tangent bundle of $M$.

ii) If $M=\{\mathrm{pt}\}$ is a point then a Lie algebroid $\mathfrak{g} \longrightarrow\{\mathrm{pt}\}$ is nothing but a Lie algebra. Thus Lie algebroids generalize Lie algebras, also.

iii) If $(M, \theta)$ is a Poisson manifold then $T^{*} M$ becomes a Lie algebroid with anchor $\varrho(\alpha)=$ $-\alpha^{\#}$ and bracket $[\alpha, \beta]_{\theta}=-\mathscr{L}_{\alpha^{\#}} \beta+\mathscr{L}_{\beta \#} \alpha-\mathrm{d}(\theta(\alpha, \beta))$, where $\alpha, \beta \in \Gamma^{\infty}\left(T^{*} M\right)$ and \# $: T^{*} M \longrightarrow T M$ is defined by $\alpha^{\#}=\theta(\cdot, \alpha)$.

One main theme in the theory of Lie algebroids is to replace the tangent bundle by $E$ and to translate geometric concepts based on the (co-)tangent bundle into the language of Lie algebroids: Indeed, for a Lie algebroid $E$, the dual bundle $E^{*}$ becomes a Poisson manifold with a Poisson bracket $\{\cdot, \cdot\}_{E}$ such that

$$
\left\{\operatorname{Pol}^{k}\left(E^{*}\right), \operatorname{Pol}^{\ell}\left(E^{*}\right)\right\}_{E} \subseteq \operatorname{Pol}^{k+\ell-1}\left(E^{*}\right),
$$

where $\operatorname{Pol}^{k}\left(E^{*}\right) \subseteq C^{\infty}\left(E^{*}\right)$ denotes those functions on $E^{*}$ which are homogeneous polynomials in fibre direction of degree $k$. This Poisson bracket is explicitly determined by

$$
\left\{\pi^{*} u, \pi^{*} v\right\}_{E}=0, \quad\left\{\pi^{*} u, \mathcal{J}(s)\right\}_{E}=\pi^{*}(\varrho(s) u), \quad \text { and } \quad\{\mathcal{J}(s), \mathcal{J}(t)\}_{E}=-\mathcal{J}\left([s, t]_{E}\right),
$$

where $\mathcal{J}: \mathcal{S}^{\bullet}(E)=\bigoplus_{k=0}^{\infty} \Gamma^{\infty}\left(\mathrm{S}^{k} E\right) \longrightarrow \operatorname{Pol}^{\bullet}\left(E^{*}\right)$ denotes the canonical graded algebra isomorphism between the symmetric $E$-tensor fields and polynomial functions on $E^{*}$. The signs in (2.2) are convention and yield the 'correct' canonical Poisson structure of $T^{*} M$ in the case of $E=T M$, 
but the negative of the usual Kirillov-Kostant-Souriau bracket on $\mathfrak{g}^{*}$ in the case of a Lie algebra $E=\mathfrak{g}$. This also motivates the notion of linear Poisson structures. The property (2.1) can equivalently be described in terms of the corresponding Poisson tensor $\theta_{E} \in \Gamma^{\infty}\left(\Lambda^{2} T E^{*}\right)$ of $\{\cdot, \cdot\}_{E}$ and the Euler vector field $\xi \in \Gamma^{\infty}\left(T E^{*}\right)$ on $E^{*}$ by

$$
\mathscr{L}_{\xi} \theta_{E}=-\theta_{E}
$$

Moreover, for $\Gamma^{\infty}\left(\Lambda^{\bullet} E^{*}\right)$ we obtain a differential $\mathrm{d}_{E}$, i.e. a super-derivation of the $\wedge$-product of degree +1 with $\mathrm{d}_{E}^{2}=0$ by literally copying the formula for the deRham differential. In particular, for $u \in C^{\infty}(M)$ we have $\left(\mathrm{d}_{E} u\right)(s)=\varrho(s) u$, and for $\alpha \in \Gamma^{\infty}\left(E^{*}\right)$ one obtains

$$
\left(\mathrm{d}_{E} \alpha\right)(s, t)=\varrho(s)(\alpha(t))-\varrho(t)(\alpha(s))-\alpha\left([s, t]_{E}\right),
$$

where $s, t \in \Gamma^{\infty}(E)$. The corresponding cohomology theory is the Lie algebroid cohomology, denoted by $\mathrm{H}_{E}^{\bullet}(M)$. In fact, all these three structures, Lie algebroid, linear Poisson structure, and differential, are completely equivalent, see e.g. [43, Section 4.2] for a pedagogical discussion.

Definition 2.2 (Gauged Poisson bracket). For an arbitrary $\mathrm{d}_{E}$-closed $E$-two-form $B_{0}$ we define the Poisson bracket $\{\cdot, \cdot\}_{B_{0}}$ by

$$
\left\{\pi^{*} u, \pi^{*} v\right\}_{B_{0}}=\left\{\pi^{*} u, \pi^{*} v\right\}_{E}=0, \quad\left\{\pi^{*} u, \mathcal{J}(s)\right\}_{B_{0}}=\left\{\pi^{*} u, \mathcal{J}(s)\right\}_{E}=\pi^{*}(\varrho(s) u),
$$

and

$$
\{\mathcal{J}(s), \mathcal{J}(t)\}_{B_{0}}=\{\mathcal{J}(s), \mathcal{J}(t)\}_{E}-\pi^{*} B_{0}(s, t)=-\mathcal{J}\left([s, t]_{E}\right)-\pi^{*} B_{0}(s, t) .
$$

We call $\{\cdot, \cdot\}_{B_{0}}$ the gauged Poisson bracket (corresponding to $B_{0}$ ).

\section{Remark 2.3.}

i) Obviously, $\{\cdot, \cdot\}_{B_{0}}$ is just a slight generalization of $\{\cdot, \cdot\}_{E}$ that coincides with this canonical Poisson bracket in case $B_{0}=0$. Observe that the $\mathrm{d}_{E}$-closedness of $B_{0}$ guarantees the Jacobi identity to be satisfied.

ii) One should note that in general the Poisson bracket $\{\cdot, \cdot\}_{B_{0}}$ does not coincide with the Poisson bracket obtained from $\theta_{E}$ via a gauge transformation $\Phi_{F}$ in the common sense (cf. [6]) which arises from a d-closed two-form $F$ on $E^{*}$. On the one hand there are Poisson brackets obtained from $\Phi_{F}\left(\theta_{E}\right)$ that are not of the particular form $\{\cdot, \cdot\}_{B_{0}}$ but on the other hand there are even Poisson brackets $\{\cdot, \cdot\}_{B_{0}}$ that cannot be obtained via such gauge transformations. For instance consider the Lie algebroid with $[\cdot, \cdot]_{E}=0$, then clearly $\theta_{E}=0$ and hence for all $F$ the Poisson tensor $\Phi_{F}\left(\theta_{E}\right)$ vanishes, but in contrast every $E$ two-form $B_{0} \neq 0$ is $\mathrm{d}_{E}$-closed and hence yields a non-vanishing Poisson bracket $\{\cdot, \cdot\}_{B_{0}}$. Another in some sense opposite extreme case occurs in case $E=T M$ with the canonical bracket, then it is easy to see that $\Phi_{F}\left(\theta_{E}\right)$ yields a bracket of the form $\{\cdot, \cdot\}_{B_{0}}$ iff $F=\pi^{*} B_{0}$.

In the following we shall make use of covariant derivatives. The adapted notion of a covariant derivative in the context of Lie algebroids is an E-connection, i.e. a bilinear map $\nabla: \Gamma^{\infty}(E) \times$ $\Gamma^{\infty}(E) \longrightarrow \Gamma^{\infty}(E)$ such that $\nabla_{s} t$ is $C^{\infty}(M)$-linear in $s$ and satisfies the Leibniz rule $\nabla_{s}(u t)=$ $(\varrho(s) u) t+u \nabla_{s} t$, where $u \in C^{\infty}(M)$ and $s, t \in \Gamma^{\infty}(E)$. For a detailed study of $E$-connections see $[20]$.

Example 2.4. Let $\nabla^{E}$ be a linear connection for $E$. Then $\nabla_{s} t=\nabla_{\varrho(s)}^{E} t$ is an $E$-connection. 
By compatibility with natural pairing and tensor products, $\nabla$ extends to all tensor powers of $E$ and $E^{*}$ in the usual way. On functions we set $\nabla_{s} u=\varrho(s) u$. The torsion $T \in \Gamma^{\infty}\left(E \otimes \Lambda^{2} E^{*}\right)$ is defined by

$$
T(s, t)=\nabla_{s} t-\nabla_{t} s-[s, t]_{E}
$$

and the curvature $R \in \Gamma^{\infty}\left(\operatorname{End}(E) \otimes \Lambda^{2} E^{*}\right)$ is defined by

$$
R(s, t)=\nabla_{s} \nabla_{t}-\nabla_{t} \nabla_{s}-\nabla_{[s, t]_{E}}
$$

as usual. If the torsion $T$ is non-trivial, one can pass to a new $E$-connection by subtracting $\frac{1}{2} T$ to obtain a torsion-free $E$-connection. Note however, that if the $E$-connection is of the form as in Example 2.4, then the result will in general no longer be of this form.

Finally, we sometimes make use of local formulas. By $e_{1}, \ldots, e_{N} \in \Gamma^{\infty}\left(\left.E\right|_{U}\right)$ we denote a local basis of sections, defined on some suitable open subset $U \subseteq M$. Then $e^{1}, \ldots, e^{N} \in \Gamma^{\infty}\left(\left.E^{*}\right|_{U}\right)$ denotes the corresponding dual basis. We have induced linear fibre coordinates $v^{\alpha}=\mathcal{J}\left(e^{\alpha}\right) \in$ $C^{\infty}\left(\left.E\right|_{U}\right)$ and $p_{\alpha}=\mathcal{J}\left(e_{\alpha}\right) \in C^{\infty}\left(\left.E^{*}\right|_{U}\right)$. If in addition $x^{1}, \ldots, x^{n}$ are local coordinates on $U$ then the anchor and the bracket are determined by

$$
\left.\varrho\right|_{U}=\varrho_{\alpha}^{i} e^{\alpha} \otimes \frac{\partial}{\partial x^{i}} \quad \text { and } \quad\left[e_{\alpha}, e_{\beta}\right]_{E}=c_{\alpha \beta}^{\gamma} e_{\gamma}
$$

with locally defined functions $\varrho_{\alpha}^{i}, c_{\alpha \beta}^{\gamma} \in C^{\infty}(U)$, respectively. Together with the fibre coordinates $p_{1}, \ldots, p_{N}$ the functions $q^{1}=\pi^{*} x^{1}, \ldots, q^{n}=\pi^{*} x^{n}$ provide a system of local coordinates on $\pi^{-1}(U) \subseteq E^{*}$. Then the Poisson tensor is locally given by

$$
\left.\theta_{E}\right|_{\pi^{-1}(U)}=\pi^{*} \varrho_{\alpha}^{i} \frac{\partial}{\partial q^{i}} \wedge \frac{\partial}{\partial p_{\alpha}}-\frac{1}{2} p_{\gamma} \pi^{*} c_{\alpha \beta}^{\gamma} \frac{\partial}{\partial p_{\alpha}} \wedge \frac{\partial}{\partial p_{\beta}}
$$

and the Hamiltonian vector field $X_{f}=\llbracket f, \theta_{E} \rrbracket$ of $f \in C^{\infty}\left(E^{*}\right)$ is

$$
\left.X_{f}\right|_{\pi^{-1}(U)}=-\pi^{*} \varrho_{\alpha}^{i} \frac{\partial f}{\partial q^{i}} \frac{\partial}{\partial p_{\alpha}}+\pi^{*} \varrho_{\alpha}^{i} \frac{\partial f}{\partial p_{\alpha}} \frac{\partial}{\partial q^{i}}+p_{\gamma} \pi^{*} c_{\alpha \beta}^{\gamma} \frac{\partial f}{\partial p_{\alpha}} \frac{\partial}{\partial p_{\beta}} .
$$

Given an $E$-connection $\nabla$ we locally have $\nabla_{e_{\alpha}} e_{\beta}=\Gamma_{\alpha \beta}^{\gamma} e_{\gamma}$ with Christoffel symbols $\Gamma_{\alpha \beta}^{\gamma} \in$ $C^{\infty}(U)$. Then $\nabla$ is torsion-free iff locally $\Gamma_{\alpha \beta}^{\gamma}-\Gamma_{\beta \alpha}^{\gamma}=c_{\alpha \beta}^{\gamma}$. For a section $s \in \Gamma^{\infty}(E)$ we have a horizontal lift $s^{\text {hor }} \in \Gamma^{\infty}\left(T E^{*}\right)$ to $E^{*}$, locally given by

$$
\left.s^{\text {hor }}\right|_{\pi^{-1}(U)}=\pi^{*}\left(s^{\alpha} \varrho_{\alpha}^{i}\right) \frac{\partial}{\partial q^{i}}+p_{\gamma} \pi^{*}\left(s^{\alpha} \Gamma_{\alpha \beta}^{\gamma}\right) \frac{\partial}{\partial p_{\beta}} .
$$

Moreover, lifting horizontally is compatible with multiplication by functions in the sense that $(u s)^{\text {hor }}=\left(\pi^{*} u\right) s^{\text {hor }}$. The section id $\in \Gamma^{\infty}(\operatorname{End}(E)) \cong \Gamma^{\infty}\left(E \otimes E^{*}\right)$ can now be lifted to a bivector field id ${ }^{\text {lift }} \in \Gamma^{\infty}\left(\otimes^{2} T E^{*}\right)$ by lifting the $E^{*}$-part vertically and the $E$-part horizontally, i.e. locally id $^{\text {lift }}=\left(e_{\alpha}\right)^{\text {hor }} \otimes\left(e^{\alpha}\right)^{\text {ver }}$. For the linear Poisson structure on $E^{*}$ we have

$$
\theta_{E}=\left(e_{\alpha}\right)^{\text {hor }} \wedge\left(e^{\alpha}\right)^{\text {ver }},
$$

i.e. the anti-symmetric part of id ${ }^{\text {lift }}$ is $\theta_{E}$. Note that $\theta_{E}$ does not depend on the choice of $\nabla$ but id $^{\text {lift }}$ does. Analogously, one has

$$
\theta_{B_{0}}=\theta_{E}-B_{0}^{\text {ver }}=\left(e_{\alpha}\right)^{\text {hor }} \wedge\left(e^{\alpha}\right)^{\text {ver }}-B_{0}^{\text {ver }}
$$

for the Poisson tensor corresponding to the gauged Poisson bracket $\{\cdot, \cdot\}_{B_{0}}$. This simple observation will be crucial for the Fedosov construction of a star product on $E^{*}$. 


\section{The Fedosov construction}

The aim of this section is to obtain deformation quantizations for the linear Poisson bracket on $E^{*}$ and the Poisson brackets arising from gauge transformations by $\mathrm{d}_{E}$-closed $E$-two forms in a geometric fashion using a variant of Fedosov's construction. Since Fedosov's construction is by now a very well-known approach to deformation quantization, which has been adapted to many contexts as e.g. [27, 5, 4, 3, 39], we can be brief. The version we are interested in resembles very much the approach of $[4$, Section 3] for cotangent bundles.

Since a star product on $E^{*}$ is completely fixed by its values on $\operatorname{Pol}^{\bullet}\left(E^{*}\right)$ and since $\operatorname{Pol}^{\bullet}\left(E^{*}\right) \cong$ $\mathcal{S}^{\bullet}(E)$ via $\mathcal{J}$, we will construct a deformation of the latter. We consider

$$
\mathcal{W}^{\bullet} \otimes \mathcal{S}^{\bullet} \otimes \Lambda^{\bullet}=\prod_{k=0}^{\infty} \bigoplus_{\ell=0}^{\infty} \Gamma^{\infty}\left(\mathrm{S}^{k} E^{*} \otimes \mathrm{S}^{\ell} E \otimes \Lambda^{\bullet} E^{*}\right)[[\lambda]],
$$

where we shall always use the sections of the complexified bundles in view of applications in physics. Note however, that in the following replacing the combination $\mathrm{i} \lambda$ by $\nu$ yields an entirely real construction in the rescaled formal parameter $\nu$. Then $\mathcal{W} \otimes \mathcal{S} \otimes \Lambda$ is an associative $\mathbb{C}[[\lambda]]$ algebra with respect to the symmetric/anti-symmetric tensor product $\mu$.

The three gradings in (3.1) give rise to degree derivations which we denote by $\operatorname{deg}_{\mathrm{s}}$ for the $\mathrm{S}^{\bullet} E^{*}$-degree, $\operatorname{deg}_{\mathrm{ver}}$ for the $\mathrm{S}^{\bullet} E$-degree, and $\operatorname{deg}_{\mathrm{a}}$ for the $\Lambda^{\bullet} E^{*}$-degree, respectively. Moreover, we have the $\lambda$-degree $\operatorname{deg}_{\lambda}=\lambda \frac{\partial}{\partial \lambda}$. Using the symmetric and anti-symmetric insertion derivations we locally have

$$
\operatorname{deg}_{\mathrm{s}}=\left(e^{\alpha} \otimes 1 \otimes 1\right) \mathrm{i}_{\mathrm{s}}\left(e_{\alpha}\right), \quad \operatorname{deg}_{\mathrm{ver}}=\left(1 \otimes e_{\alpha} \otimes 1\right) \mathrm{i}_{\mathrm{s}}\left(e^{\alpha}\right), \quad \text { and } \quad \operatorname{deg}_{\mathrm{a}}=\left(1 \otimes 1 \otimes e^{\alpha}\right) \mathrm{i}_{\mathrm{a}}\left(e_{\alpha}\right) .
$$

Moreover, we shall use the total degree $\operatorname{Deg}=\operatorname{deg}_{\mathrm{s}}+\operatorname{deg}_{\lambda}$ as well as the homogeneity operator (or: $\lambda$-Euler derivation) $\mathcal{H}=\operatorname{deg}_{\text {ver }}+\operatorname{deg}_{\lambda}$. The product $\mu$ of $\mathcal{W}^{\bullet} \otimes \mathcal{S}^{\bullet} \otimes \Lambda^{\bullet}$ is graded with respect to all degree maps, i.e. $\operatorname{deg}_{\mathrm{s}}, \operatorname{deg}_{\mathrm{a}}, \operatorname{deg}_{\mathrm{ver}}, \operatorname{deg}_{\lambda}$ and hence Deg and $\mathcal{H}$ are derivations. Moreover, $\mu$ is super-commutative with respect to $\operatorname{deg}_{a}$. The space of elements in $\mathcal{W} \otimes \mathcal{S} \otimes \Lambda$ of total degree $\geq k$ will be denoted by $\mathcal{W}_{k} \otimes \mathcal{S} \otimes \Lambda$.

As usual in the Fedosov framework we consider the maps

$$
\delta=\left(1 \otimes 1 \otimes e^{\alpha}\right) \mathrm{i}_{\mathrm{s}}\left(e_{\alpha}\right) \quad \text { and } \quad \delta^{*}=\left(e^{\alpha} \otimes 1 \otimes 1\right) \mathrm{i}_{\mathrm{a}}\left(e_{\alpha}\right)
$$

together with $\delta^{-1}$, defined by $\delta^{-1} a=\frac{1}{k+\ell} a$ for $\operatorname{deg}_{\mathrm{s}} a=k a$ and $\operatorname{deg}_{\mathrm{a}} a=\ell a$ if $k+\ell>0$ and $\delta^{-1} a=0$ otherwise. Moreover,

$$
\sigma: \mathcal{W} \otimes \mathcal{S} \otimes \Lambda \longrightarrow \mathcal{S}
$$

denotes the projection onto the part of $\operatorname{deg}_{s^{-}}$and $\operatorname{deg}_{a}$-degree 0 . Then one has

$$
\delta^{2}=0, \quad\left(\delta^{-1}\right)^{2}=0 \quad \text { and } \quad \delta \delta^{-1}+\delta^{-1} \delta+\sigma=\mathrm{id} .
$$

The degrees of the maps $\delta$ and $\delta^{-1}$ are

$$
\left[\operatorname{deg}_{\mathrm{s}}, \delta\right]=-\delta, \quad\left[\operatorname{deg}_{\mathrm{a}}, \delta\right]=\delta, \quad \text { and } \quad\left[\operatorname{deg}_{\lambda}, \delta\right]=0=\left[\operatorname{deg}_{\mathrm{ver}}, \delta\right]
$$

as well as

$$
\left[\operatorname{deg}_{\mathrm{s}}, \delta^{-1}\right]=\delta^{-1}, \quad\left[\operatorname{deg}_{\mathrm{a}}, \delta^{-1}\right]=-\delta^{-1}, \quad \text { and } \quad\left[\operatorname{deg}_{\lambda}, \delta^{-1}\right]=0=\left[\operatorname{deg}_{\mathrm{ver}}, \delta^{-1}\right] .
$$

In a next step, one deforms $\mathcal{W} \otimes \mathcal{S} \otimes \Lambda$ using ' $\kappa$-ordered' deformations, explicitly given by

$$
a \circ_{\kappa} b=\mu \circ \exp \left(-(1-\kappa) \mathrm{i} \lambda \mathrm{i}_{\mathrm{s}}\left(e^{\alpha}\right) \otimes \mathrm{i}_{\mathrm{s}}\left(e_{\alpha}\right)+\kappa \mathrm{i} \lambda \mathrm{i}_{\mathrm{s}}\left(e_{\alpha}\right) \otimes \mathrm{i}_{\mathrm{s}}\left(e^{\alpha}\right)\right)(a \otimes b),
$$


where $\kappa \in \mathbb{R}$ denotes a real parameter. Note that $\circ_{\kappa}$ is globally well defined. We are mainly interested in $\kappa \in[0,1]$. Analogously to the case of cotangent bundles the products for $\kappa=0$, $\kappa=\frac{1}{2}$, and $\kappa=1$ are referred to as standard-ordered, Weyl-ordered, and anti-standard-ordered fibrewise product and are denoted by $\circ_{0}=\circ_{\text {Std }}, \circ_{\frac{1}{2}}=\circ_{\text {Weyl }}$, and $\circ_{1}=\circ_{\overline{\mathrm{Std}}}$, respectively. By the usual 'commuting derivation' argument, $\circ_{\kappa}$ is associative for all $\kappa \in \mathbb{R}$. All these deformed products quantize the same fibrewise (super-) Poisson structure $\{\cdot, \cdot\}_{\mathrm{fib}}$.

The total degree Deg, the homogeneity operator $\mathcal{H}$ as well as $\operatorname{deg}_{a}$ are still derivations of $\circ_{\kappa}$. Moreover,

$$
\delta=\frac{\mathrm{i}}{\lambda} \operatorname{ad}_{\kappa}\left(1 \otimes \mathrm{id}_{E}\right),
$$

where we view $\mathrm{id}_{E} \in \Gamma^{\infty}(\operatorname{End}(E))$ as an element in $\mathcal{W}^{0} \otimes \mathcal{S}^{1} \otimes \Lambda^{1}$ and $\operatorname{ad}_{\kappa}$ denotes the $\circ_{\kappa}$-supercommutator. Thus $\delta$ is a quasi-inner derivation and clearly $\delta\left(1 \otimes \mathrm{id}_{E}\right)=0$.

Considering the fibrewise 'Laplacian' $\Delta_{\text {fib }}$ defined by

$$
\Delta_{\mathrm{fib}}=\mathrm{i}_{\mathrm{s}}\left(e^{\alpha}\right) \mathrm{i}_{\mathrm{s}}\left(e_{\alpha}\right)
$$

we have the identity

$$
\Delta_{\mathrm{fib}} \circ \mu=\mu \circ\left(\Delta_{\mathrm{fib}} \otimes \mathrm{id}+\mathrm{i}_{\mathrm{s}}\left(e^{\alpha}\right) \otimes \mathrm{i}_{\mathrm{s}}\left(e_{\alpha}\right)+\mathrm{i}_{\mathrm{s}}\left(e_{\alpha}\right) \otimes \mathrm{i}_{\mathrm{s}}\left(e^{\alpha}\right)+\mathrm{id} \otimes \Delta_{\mathrm{fib}}\right) .
$$

With this equation one can easily show that

$$
\mathcal{M}_{\kappa^{\prime}-\kappa}=\exp \left(\mathrm{i} \lambda\left(\kappa^{\prime}-\kappa\right) \Delta_{\mathrm{fib}}\right)
$$

defines a fibrewise equivalence transformation from $\left(\mathcal{W} \otimes \mathcal{S} \otimes \Lambda, \circ_{\kappa}\right)$ to $\left(\mathcal{W} \otimes \mathcal{S} \otimes \Lambda, \circ_{\kappa^{\prime}}\right)$. Note that we have the commutation relations

$$
\left[\delta, \Delta_{\mathrm{fib}}\right]=\left[\operatorname{deg}_{\lambda}, \Delta_{\mathrm{fib}}\right]=\left[\operatorname{deg}_{\mathrm{a}}, \Delta_{\mathrm{fib}}\right]=0 \quad \text { and } \quad\left[\operatorname{deg}_{\mathrm{s}}, \Delta_{\mathrm{fib}}\right]=\left[\operatorname{deg}_{\mathrm{ver}}, \Delta_{\mathrm{fib}}\right]=-\Delta_{\mathrm{fib}} \text {. }
$$

The super-centre of $o_{\kappa}$ is described as follows:

Lemma 3.1. $\operatorname{ad}_{\kappa}(a)=0$ iff $\operatorname{deg}_{\mathrm{s}} a=0=\operatorname{deg}_{\mathrm{ver}} a$, i.e. $a \in \Gamma^{\infty}\left(\Lambda^{\bullet} E^{*}\right)[[\lambda]]$.

Now we choose a torsion-free $E$-connection $\nabla$ which gives an exterior covariant derivative

$$
D=\left(1 \otimes 1 \otimes e^{\alpha}\right) \nabla_{e_{\alpha}}
$$

Then $D$ is clearly globally well defined. Moreover, and this is remarkable compared to the usual Fedosov approach, $D$ is a super-derivation of $\circ_{\kappa}$ without any further assumptions. This is clear, as $\circ_{\kappa}$ only involves natural pairings. The properties of $D$ are easily determined and will be summarized in the following lemma:

Lemma 3.2. $D$ is a super-derivation of $\circ_{\kappa}$ with $\left[\operatorname{deg}_{\mathrm{s}}, D\right]=\left[\operatorname{deg}_{\lambda}, D\right]=\left[\operatorname{deg}_{\mathrm{ver}}, D\right]=0,\left[\operatorname{deg}_{\mathrm{a}}, D\right]$ $=D$, and $\left[\Delta_{\mathrm{fib}}, D\right]=0$. Moreover,

$$
D^{2}=\frac{1}{2}[D, D]=-\frac{\mathrm{i}}{\lambda} \operatorname{ad}_{\kappa}(R) \quad \text { and } \quad[\delta, D]=0 .
$$

The curvature $R \in \mathcal{W}^{1} \otimes \mathcal{S}^{1} \otimes \Lambda^{2}$ satisfies the Bianchi identities $\delta R=0=D R$. Moreover, $\Delta_{\mathrm{fib}} R \in \Gamma^{\infty}\left(\Lambda^{2} E^{*}\right)$ is exact, i.e. there is an E-one-form $\Gamma$ such that $-\Delta_{\mathrm{fib}} R=\mathrm{d}_{E} \Gamma$. Finally, for $B \in \Gamma^{\infty}\left(\Lambda^{\bullet} E^{*}\right)$ we have $D B=\mathrm{d}_{E} B$. 
Note that the lemma allows for some rather obvious modifications in case the $E$-connection has torsion analogous to $[28,37]$.

Now, one makes the usual ansatz

$$
\mathcal{D}_{\kappa}=-\delta+D+\frac{\mathrm{i}}{\lambda} \operatorname{ad}_{\kappa}\left(r_{\kappa}\right)
$$

with an element $r_{\kappa} \in \mathcal{W}_{1} \otimes \mathcal{S} \otimes \Lambda^{1}$. Clearly, $\mathcal{D}_{\kappa}$ is a super-derivation of $\circ_{\kappa}$, and we want to find $r_{\kappa}$ such that $\mathcal{D}_{\kappa}^{2}=0$. A direct computation yields that

$$
\mathcal{D}_{\kappa}^{2}=\frac{\mathrm{i}}{\lambda} \operatorname{ad}_{\kappa}\left(-\delta r_{\kappa}+D r_{\kappa}+\frac{\mathrm{i}}{\lambda} r_{\kappa} \circ_{\kappa} r_{\kappa}-R\right),
$$

which vanishes iff $-\delta r_{\kappa}+D r_{\kappa}+\frac{\mathrm{i}}{\lambda} r_{\kappa} \circ_{\kappa} r_{\kappa}-R$ is a central element in $\mathcal{W} \otimes \mathcal{S} \otimes \Lambda^{2}$. This is the case iff there is a formal series of two-forms $B \in \Gamma^{\infty}\left(\Lambda^{2} E^{*}\right)[[\lambda]]$ with

$$
\delta r_{\kappa}-D r_{\kappa}-\frac{\mathrm{i}}{\lambda} r_{\kappa} \circ_{\kappa} r_{\kappa}+R=B .
$$

Since $\left[\mathcal{D}_{\kappa},\left[\mathcal{D}_{\kappa}, \mathcal{D}_{\kappa}\right]\right]=0$ by the super-Jacobi identity the necessary condition for this equation to be solvable is $\mathcal{D}_{\kappa} B=\mathrm{d}_{E} B=0$.

After these preparations one is in the position to prove the following theorem in analogy to [17, Theorem 3.2] and [18, Theorem 5.2.2]:

Theorem 3.3. For every formal series $B=\sum_{j=0}^{\infty} \lambda^{j} B_{j} \in \Gamma^{\infty}\left(\Lambda^{2} E^{*}\right)[[\lambda]]$ of $\mathrm{d}_{E}$-closed E-twoforms there exists a unique element $r_{\kappa} \in \mathcal{W}_{1} \otimes \mathcal{S} \otimes \Lambda^{1}$ such that

$$
\delta r_{\kappa}=D r_{\kappa}+\frac{\mathrm{i}}{\lambda} r_{\kappa} \circ_{\kappa} r_{\kappa}-R+B \quad \text { and } \quad \delta^{-1} r_{\kappa}=0 .
$$

Moreover, $r_{\kappa}$ satisfies

$$
r_{\kappa}=\delta^{-1}\left(D r_{\kappa}+\frac{\mathrm{i}}{\lambda} r_{\kappa} \circ_{\kappa} r_{\kappa}-R+B\right)
$$

from which $r_{\kappa}$ can be determined recursively. In this case the Fedosov derivation

$$
\mathcal{D}_{\kappa}=-\delta+D+\frac{\mathrm{i}}{\lambda} \operatorname{ad}_{\kappa}\left(r_{\kappa}\right)
$$

is a $\circ_{\kappa}$-super-derivation of $\Lambda^{\bullet} E^{*}$-degree +1 and has square zero: $\mathcal{D}_{\kappa}^{2}=0$.

The proof follows the standard line of argument with the only exception that thanks to our particular gradings also $B_{0} \neq 0$ is possible.

Before investigating the structure of $\operatorname{ker}\left(\mathcal{D}_{\kappa}\right) \cap \mathcal{W} \otimes \mathcal{S}$ we note that the $\mathcal{D}_{\kappa}$-cohomology is trivial on elements $a$ with positive $\Lambda^{\bullet} E^{*}$-degree since one has the following homotopy formula

$$
\mathcal{D}_{\kappa} \mathcal{D}_{\kappa}^{-1} a+\mathcal{D}_{\kappa}^{-1} \mathcal{D}_{\kappa} a+\frac{1}{\mathrm{id}-\left[\delta^{-1}, D+\frac{\mathrm{i}}{\lambda} \operatorname{ad}_{\kappa}\left(r_{\kappa}\right)\right]} \sigma(a)=a,
$$

where

$$
\mathcal{D}_{\kappa}^{-1} a=-\delta^{-1}\left(\frac{1}{\mathrm{id}-\left[\delta^{-1}, D+\frac{\mathrm{i}}{\lambda} \operatorname{ad}_{\kappa}\left(r_{\kappa}\right)\right]} a\right)
$$

for all $a \in \mathcal{W} \otimes \mathcal{S} \otimes \Lambda$ (cf. [18, Theorem 5.2.5]). Clearly, (3.7) is a deformation of (3.2).

The next step in Fedosov's construction now consists in establishing a bijection between the elements $a$ of $\mathcal{W} \otimes \mathcal{S}$ with $\mathcal{D}_{\kappa} a=0$ and $\mathcal{S}$, which can be done as in [17, Theorem 3.3] or [18, Theorem 5.2.4]. 
Theorem 3.4. Let $\mathcal{D}_{\kappa}=-\delta+D+\frac{\mathrm{i}}{\lambda} \operatorname{ad}_{\kappa}\left(r_{\kappa}\right): \mathcal{W} \otimes \mathcal{S} \otimes \Lambda^{\bullet} \longrightarrow \mathcal{W} \otimes \mathcal{S} \otimes \Lambda^{\bullet+1}$ be given as in (3.6) with $r_{\kappa}$ as in (3.4).

i) Then for any $s \in \mathcal{S}=\mathcal{S}^{\bullet}(E)[[\lambda]]=\bigoplus_{\ell=0}^{\infty} \Gamma^{\infty}\left(\mathrm{S}^{\ell} E\right)[[\lambda]]$ there exists a unique element $\tau_{\kappa}(s) \in \operatorname{ker}\left(\mathcal{D}_{\kappa}\right) \cap \mathcal{W} \otimes \mathcal{S}$ such that

$$
\sigma\left(\tau_{\kappa}(s)\right)=s
$$

and $\tau_{\kappa}: \mathcal{S} \longrightarrow \operatorname{ker}\left(\mathcal{D}_{\kappa}\right) \cap \mathcal{W} \otimes \mathcal{S}$ is $\mathbb{C}[[\lambda]]$-linear and referred to as the Fedosov-Taylor series corresponding to $\mathcal{D}_{\kappa}$.

ii) In addition $\tau_{\kappa}(s)$ is given by

$$
\tau_{\kappa}(s)=\frac{1}{\mathrm{id}-\left[\delta^{-1}, D+\frac{\mathrm{i}}{\lambda} \operatorname{ad}_{\kappa}\left(r_{\kappa}\right)\right]} s
$$

or can be determined recursively from

$$
\tau_{\kappa}(s)=s+\delta^{-1}\left(D \tau_{\kappa}(s)+\frac{\mathrm{i}}{\lambda} \operatorname{ad}_{\kappa}\left(r_{\kappa}\right) \tau_{\kappa}(s)\right)
$$

iii) Since $\mathcal{D}_{\kappa}$ as constructed above is a $\circ_{\kappa}$-super-derivation and since $\operatorname{ker}\left(\mathcal{D}_{\kappa}\right) \cap \mathcal{W} \otimes \mathcal{S}$ is $a \circ_{\kappa}$-subalgebra, a new associative product $*_{\kappa}$ for $\mathcal{S}$ that deforms the symmetric product is defined by pull-back of $\mathrm{o}_{\kappa}$ via $\tau_{\kappa}$ :

$$
s *_{\kappa} t=\sigma\left(\tau_{\kappa}(s) \circ_{\kappa} \tau_{\kappa}(t)\right) .
$$

In the following we shall refer to the associative product $*_{\kappa}$ defined above as the $\kappa$-ordered $E$-Fedosov product (corresponding to $(\nabla, B)$ ).

Clearly, from the associative deformation above $\mathcal{S}^{\bullet}(E)$ inherits the structure of a Poisson algebra by the term occurring in the first order $i \lambda$ of the $*_{\kappa}$-commutator. As a first result we prove that the corresponding Poisson bracket $\{\cdot, \cdot\}_{*_{\kappa}}$ coincides with the one induced on $\mathcal{S}^{\bullet}(E)$ by pull-back of $\{\cdot, \cdot\}_{B_{0}}$ via $\mathcal{J}$.

Proposition 3.5. For all $s, t \in \mathcal{S}^{\bullet}(E)$ we have

$$
s *_{\kappa} t=s t+\mathrm{i} \lambda\left(-(1-\kappa) \mathrm{i}_{\mathrm{s}}\left(e^{\alpha}\right) s \nabla_{e_{\alpha}} t+\kappa \nabla_{e_{\alpha}} s \mathrm{i}_{\mathrm{s}}\left(e^{\alpha}\right) t-\frac{1}{2} B_{0}\left(e_{\alpha}, e_{\beta}\right) \mathrm{i}_{\mathrm{s}}\left(e^{\alpha}\right) s \mathrm{i}_{\mathrm{s}}\left(e^{\beta}\right) t\right)+O\left(\lambda^{2}\right) .
$$

In particular, this implies that the induced Poisson bracket on $\mathcal{S}^{\bullet}(E)$ is given by

$$
\{s, t\}_{*_{\kappa}}=\nabla_{e_{\alpha}} s \mathrm{i}_{\mathrm{s}}\left(e^{\alpha}\right) t-\mathrm{i}_{\mathrm{s}}\left(e^{\alpha}\right) s \nabla_{e_{\alpha}} t-B_{0}\left(e_{\alpha}, e_{\beta}\right) \mathrm{i}_{\mathrm{s}}\left(e^{\alpha}\right) s \mathrm{i}_{\mathrm{s}}\left(e^{\beta}\right) t=\mathcal{J}^{-1}\{\mathcal{J}(s), \mathcal{J}(t)\}_{B_{0}} .
$$

In case $B_{0}=0$ we therefore have $\{s, t\}_{*_{\kappa}}=\mathcal{J}^{-1}\{\mathcal{J}(s), \mathcal{J}(t)\}_{E}$.

Proof. By straightforward computation using that $\sigma$ commutes with $\mathrm{i}_{\mathrm{s}}(\alpha)$ for all $\alpha \in \Gamma^{\infty}\left(E^{*}\right)$ one finds

$$
s *_{\kappa} t=s t-(1-\kappa) \mathrm{i} \lambda \mathrm{i}_{\mathrm{s}}\left(e^{\alpha}\right) s \mathrm{i}_{\mathrm{s}}\left(e_{\alpha}\right) \tau_{\kappa}(t)_{\mathrm{cl}}^{1}+\kappa \mathrm{i} \lambda \mathrm{i}_{\mathrm{s}}\left(e_{\alpha}\right) \tau_{\kappa}(s)_{\mathrm{cl}}^{1} \mathrm{i}_{\mathrm{s}}\left(e^{\alpha}\right) t+O\left(\lambda^{2}\right),
$$

where $\tau_{\kappa}(t)_{\mathrm{cl}}^{1}$ denotes the term of $\mathrm{S}^{\bullet} E^{*}$-degree 1 of the classical part of $\tau_{\kappa}(t)$. From equation (3.8) one finds that $\tau_{\kappa}(t)_{\mathrm{cl}}^{1}$ satisfies

$$
\tau_{\kappa}(t)_{\mathrm{cl}}^{1}=\delta^{-1}\left(D t+\mathrm{i}_{\mathrm{s}}\left(e^{\alpha}\right) r_{\kappa, \mathrm{cl}}^{0} \mathrm{i}_{\mathrm{s}}\left(e_{\alpha}\right) \tau_{\kappa}(t)_{\mathrm{cl}}^{1}-\mathrm{i}_{\mathrm{s}}\left(e^{\alpha}\right) t \mathrm{i}_{\mathrm{s}}\left(e_{\alpha}\right) r_{\kappa, \mathrm{cl}}^{1}\right)
$$

where again ${ }_{\mathrm{cl}}$ refers to the classical part and the upper index indicates the $\mathrm{S}^{\bullet} E^{*}$-degree. But from (3.5) it is evident that $r_{\kappa, \mathrm{cl}}^{0}=0$ which in turn implies that $r_{\kappa, \mathrm{cl}}^{1}=\delta^{-1} B_{0}$. Therefore 
we explicitly get $\tau(t)_{\mathrm{cl}}^{1}=\delta^{-1}\left(D t-\frac{1}{2} \mathrm{i}_{\mathrm{s}}\left(e^{\beta}\right) t \mathrm{i}_{\mathrm{a}}\left(e_{\beta}\right) B_{0}\right)=\left(1 \otimes e^{\alpha}\right)\left(\nabla_{e_{\alpha}} t+\frac{1}{2} B_{0}\left(e_{\alpha}, e_{\beta}\right) \mathrm{i}_{\mathrm{s}}\left(e^{\beta}\right) t\right)$. Together with the above expression for $s *_{\kappa} t$ this implies the first statement of the proposition. For the proof of the formula for $\{s, t\}_{*_{\kappa}}$ it suffices to check the statement on the generators of $\mathcal{S}^{\bullet}(E)$, i.e. on functions $u, v \in C^{\infty}(M)$ and on $s, t \in \Gamma^{\infty}(E)$. But this is straightforward using the properties of a torsion-free $E$-connection. This fact can also directly be read off the formulas (2.4) and (2.5).

Clearly, the products $*_{\kappa}$ give rise to star products $\star_{\kappa}$ on $\mathrm{Pol}^{\bullet}\left(E^{*}\right)[[\lambda]]$ by defining

$$
f \star_{\kappa} g=\mathcal{J}\left(\mathcal{J}^{-1}(f) *_{\kappa} \mathcal{J}^{-1}(g)\right)
$$

for $f, g \in \mathrm{Pol}^{\bullet}\left(E^{*}\right)[[\lambda]]$. According to Proposition 3.5 this is a deformation in the direction of the Poisson bracket $\{\cdot, \cdot\}_{B_{0}}$. But in order to be able to extend the latter product to a star product on $C^{\infty}\left(E^{*}\right)[[\lambda]]$ one additionally has to show that on $\operatorname{Pol}^{\bullet}\left(E^{*}\right)[[\lambda]]$ the product $\star_{\kappa}$ can be described by bidifferential operators (cf. the discussion in [4, Section 3]).

Proposition 3.6. Writing the term of total degree $k \in \mathbb{N}$ in $\tau_{\kappa}(t)$ for $t \in \mathcal{S}^{\bullet}(E)=\Gamma^{\infty}\left(\mathrm{S}^{\bullet} E\right)$ as $\tau_{\kappa}(t)^{(k)}=\sum_{\ell=0}^{k}\left(\frac{\lambda}{\mathrm{i}}\right)^{k-\ell} \tau_{\kappa}(t)^{(k), \ell}$ with $\tau_{\kappa}(t)^{(k), \ell} \in \Gamma^{\infty}\left(\mathrm{S}^{\ell} E^{*} \otimes \mathrm{S}^{\bullet} E\right)$ one has that the mapping

$$
t \mapsto \tau_{\kappa}(t)^{(k), \ell}
$$

is a differential operator of order $k$ for all $0 \leq \ell \leq k$.

Proof. For the proof we use the usual algebraic definition of differential operators on appropriate subspaces of the $\mathcal{S}^{\bullet}(E)=\Gamma^{\infty}\left(\mathrm{S}^{\bullet} E\right)$ (left) module $\prod_{k=0}^{\infty} \Gamma^{\infty}\left(\mathrm{S}^{k} E^{*} \otimes \mathrm{S}^{\bullet} E \otimes \Lambda^{\bullet} E^{*}\right)$ that take their values again in some subspace of $\prod_{k=0}^{\infty} \Gamma^{\infty}\left(S^{k} E^{*} \otimes S^{\bullet} E \otimes \Lambda^{\bullet} E^{*}\right)$, see e.g. [43]. Clearly, this definition also applies to differential operators on the algebra $\mathcal{S}^{\bullet}(E)$ with values in some subspace of the $\mathcal{S}^{\bullet}(E)$ (left) module $\prod_{k=0}^{\infty} \Gamma^{\infty}\left(\mathrm{S}^{k} E^{*} \otimes \mathrm{S}^{\bullet} E \otimes \Lambda^{\bullet} E^{*}\right)$. With this notion it is evident that $\delta^{-1}$ is a differential operator of order 0 since it commutes with all (left) multiplications by elements of $\mathcal{S}^{\bullet}(E)$. Likewise, $\mathrm{i}_{\mathrm{s}}(s)$ is a differential operator of order 0 for all $s \in \Gamma^{\infty}(E)$. Furthermore, $D$ as well as $\mathrm{i}_{\mathrm{s}}(\alpha)$ for $\alpha \in \Gamma^{\infty}\left(E^{*}\right)$ are differential operators of order 1 since the respective commutators with (left) multiplications by elements of $\mathcal{S}^{\bullet}(E)$ are given by (left) multiplications, which are by definition differential operators of order 0 . Moreover, we use the well-known fact that composing two differential operators of order $k$ and $k^{\prime}$ the result is a differential operator of order $k+k^{\prime}$. After these preparations the proof of the proposition is a rather straightforward induction on the total degree. It is trivial that $t \mapsto \tau_{\kappa}(t)^{(0)}=t$ is a differential operator of order 0 . Now let us assume that we have shown that for $j=0, \ldots, k$ we have that $t \mapsto \tau_{\kappa}(t)^{(j)}$ consists of differential operators of order $j$. Using the recursion formula (3.8) for $\tau_{\kappa}(t)$ and observing the respective total degree of the involved elements it is lengthy but straightforward to show that

$$
\begin{aligned}
& \tau_{\kappa}(t)^{(k+1)}=\delta^{-1} D \tau_{\kappa}(t)^{(k)} \\
& +\delta^{-1}\left(\sum_{\ell=0}^{k} \sum_{j=0}^{k-\ell} \sum_{m=1}^{\ell+1}\left(\frac{\lambda}{\mathrm{i}}\right)^{j+m-1} \frac{(1-\kappa)^{j}(-\kappa)^{m}}{j ! m !} \mathrm{i}_{\mathrm{S}}\left(e^{\alpha_{1}}\right) \cdots \mathrm{i}_{\mathrm{s}}\left(e^{\alpha_{j}}\right) \mathrm{i}_{\mathrm{S}}\left(e_{\beta_{1}}\right) \cdots \mathrm{i}_{\mathrm{s}}\left(e_{\beta_{m}}\right) r_{\kappa}^{(\ell+1)}\right. \\
& \left.\quad \mathrm{i}_{\mathrm{S}}\left(e_{\alpha_{1}}\right) \cdots \mathrm{i}_{\mathrm{S}}\left(e_{\alpha_{j}}\right) \mathrm{i}_{\mathrm{S}}\left(e^{\beta_{1}}\right) \cdots \mathrm{i}_{\mathrm{S}}\left(e^{\beta_{m}}\right) \tau_{\kappa}(t)^{(k-\ell)}\right) \\
& -\delta^{-1}\left(\sum_{\ell=0}^{k} \sum_{j=1}^{\ell+1} \sum_{m=0}^{k-\ell}\left(\frac{\lambda}{\mathrm{i}}\right)^{j+m-1} \frac{(1-\kappa)^{j}(-\kappa)^{m}}{j ! m !} \mathrm{i}_{\mathrm{S}}\left(e^{\alpha_{1}}\right) \cdots \mathrm{i}_{\mathrm{S}}\left(e^{\alpha_{j}}\right) \mathrm{i}_{\mathrm{s}}\left(e_{\beta_{1}}\right) \cdots \mathrm{i}_{\mathrm{S}}\left(e_{\beta_{m}}\right) \tau_{\kappa}(t)^{(k-\ell)}\right. \\
& \left.\quad \mathrm{i}_{\mathrm{S}}\left(e_{\alpha_{1}}\right) \cdots \mathrm{i}_{\mathrm{S}}\left(e_{\alpha_{j}}\right) \mathrm{i}_{\mathrm{S}}\left(e^{\beta_{1}}\right) \cdots \mathrm{i}_{\mathrm{S}}\left(e^{\beta_{m}}\right) r_{\kappa}^{(\ell+1)}\right) .
\end{aligned}
$$


Since $\delta^{-1}$ is a differential operator of order 0 and $D$ is a differential operator of order 1 the induction hypotheses yields that $t \mapsto \delta^{-1} D \tau_{\kappa}(t)^{(k)}$ only contains differential operators of order at most $k+1$. Furthermore, from the fact that $t \mapsto \mathrm{i}_{\mathrm{S}}\left(e^{\beta_{1}}\right) \cdots \mathrm{i}_{\mathrm{S}}\left(e^{\beta_{m}}\right) \tau_{\kappa}(t)^{(k-\ell)}$ only contains differential operators of order at most $k-\ell+m$ and observing that the sum over $m$ in the second summand runs from 1 to $\ell+1$ the order of the differential operators occurring in the second term is at most $k+1$. Here we additionally have used that multiplications with elements in $\prod_{k=0}^{\infty} \Gamma^{\infty}\left(S^{k} E^{*} \otimes S^{\bullet} E \otimes \Lambda^{\bullet} E^{*}\right)$ are of order 0 and that the symmetric insertions of sections in $E$ are of order 0 also. The same line of argument applied to the third summand in $\tau_{\kappa}(t)^{(k+1)}$ yields that this part of the Fedosov Taylor series only consists in differential operators of order $k+1$ proving the assertion by induction.

After these preparations we can state the main result of this section:

Theorem 3.7. For all $\kappa \in \mathbb{R}$ and all $\mathrm{d}_{E}$-closed $B \in \Gamma^{\infty}\left(\Lambda^{2} E^{*}\right)[[\lambda]]$ one has:

i) For all $s, t \in \mathcal{S}^{\bullet}(E)$ the product $*_{\kappa}$ can be written as

$$
s *_{\kappa} t=\sum_{k=0}^{\infty} \lambda^{k} D_{k}(s, t)
$$

where $D_{k}: \mathcal{S}^{\bullet}(E) \times \mathcal{S}^{\bullet}(E) \longrightarrow \mathcal{S}^{\bullet}(E)$ is a bidifferential operator of order $k$ in both arguments.

ii) For all $f, g \in \mathrm{Pol}^{\bullet}\left(E^{*}\right)$ the product $\star_{\kappa}$ is given by

$$
f \star_{\kappa} g=\sum_{k=0}^{\infty} \lambda^{k} C_{k}(f, g)=\sum_{k=0}^{\infty} \lambda^{k} \mathcal{J}\left(D_{k}\left(\mathcal{J}^{-1}(f), \mathcal{J}^{-1}(g)\right)\right),
$$

where $C_{k}: \mathrm{Pol}^{\bullet}\left(E^{*}\right) \times \mathrm{Pol}^{\bullet}\left(E^{*}\right) \longrightarrow \mathrm{Pol}^{\bullet}\left(E^{*}\right)$ is a bidifferential operator of order $k$ in both arguments. Hence $\star_{\kappa}$ extends in a unique way to a star product on $C^{\infty}\left(E^{*}\right)[[\lambda]]$ with respect to the Poisson bracket $\{\cdot, \cdot\}_{B_{0}}$ which is in addition natural in the sense of [25].

Proof. For the proof of the first statement an easy computation expressing $s *_{\kappa} t$ by means of $\tau_{\kappa}(s)^{(m), \ell}$ and $\tau_{\kappa}(t)^{(k-m), \ell^{\prime}}$ as in Proposition 3.6 yields

$$
\begin{gathered}
D_{k}(s, t)=(-\mathrm{i})^{k} \sum_{m=0}^{k} \sum_{\ell^{\prime}=0}^{k-m} \sum_{\ell=0}^{m} \frac{(1-\kappa)^{\ell^{\prime}}(-\kappa)^{\ell}}{\ell ! \ell^{\prime} !} \mathrm{i}_{\mathrm{s}}\left(e^{\alpha_{1}}\right) \cdots \mathrm{i}_{\mathrm{s}}\left(e^{\alpha_{\ell^{\prime}}}\right) \mathrm{i}_{\mathrm{s}}\left(e_{\beta_{1}}\right) \cdots \mathrm{i}_{\mathrm{s}}\left(e_{\beta_{\ell}}\right) \tau_{\kappa}(s)^{(m), \ell} \\
\mathrm{i}_{\mathrm{S}}\left(e^{\beta_{1}}\right) \cdots \mathrm{i}_{\mathrm{s}}\left(e^{\beta_{\ell}}\right) \mathrm{i}_{\mathrm{s}}\left(e_{\alpha_{1}}\right) \cdots \mathrm{i}_{\mathrm{s}}\left(e_{\alpha_{\ell^{\prime}}}\right) \tau_{\kappa}(t)^{(k-m), \ell^{\prime}} .
\end{gathered}
$$

Now according to Proposition 3.6 the map $s \mapsto \mathrm{i}_{\mathrm{S}}\left(e^{\alpha_{1}}\right) \cdots \mathrm{i}_{\mathrm{S}}\left(e^{\alpha_{\ell^{\prime}}}\right) \tau_{\kappa}(s)^{(m), \ell}$ is a differential operator of order $m+\ell^{\prime}$ and $t \mapsto \mathrm{i}_{\mathrm{s}}\left(e^{\beta_{1}}\right) \cdots \mathrm{i}_{\mathrm{s}}\left(e^{\beta_{\ell}}\right) \tau_{\kappa}(t)^{(k-m), \ell^{\prime}}$ is a differential operator of order $k-m+\ell$. Observing that the sum over $\ell^{\prime}$ runs from 0 to $k-m$ and that the sum over $\ell$ runs from 0 to $m$ it is then obvious that the highest order of differentiation occurring in $D_{k}(s, t)$ is $k$ in both arguments proving $i$ ). The first part of the second statement is trivial since $\mathcal{J}$ is an isomorphism between the undeformed associative algebras $\mathcal{S}^{\bullet}(E)$ and $\operatorname{Pol}^{\bullet}\left(E^{*}\right)$. The last part of the second statement follows from the fact that bidifferential operators on $C^{\infty}\left(E^{*}\right)$ are completely determined by their values on $\operatorname{Pol}^{\bullet}\left(E^{*}\right)$.

Remark 3.8. Because of the statements of the preceding theorem it is sufficient to prove properties of $\star_{\kappa}$ on $\mathrm{Pol}^{\bullet}\left(E^{*}\right)$ in order to show that they actually hold on all of $C^{\infty}\left(E^{*}\right)$. This observation drastically simplifies many of the further investigations in the following sections. 


\section{Further properties of $\star_{\kappa}$}

In this section we shall first find necessary conditions on the data determining the star products $\star_{\kappa}$ that guarantee that these products are homogeneous in the sense of Definition 1.1. These star products are of particular interest since the results of Section 7 about the trace functional apply. Moreover, we will show that the recursion formula for the element $r_{\kappa}$ determining the Fedosov derivation drastically simplifies due to the special shape of the fibrewise product $\circ_{\kappa}$ and provides some rather explicit formulas. In particular, we explicitly compute the star products of an arbitrary function $f \in C^{\infty}\left(E^{*}\right)$ with the pull-back $\pi^{*} u$ of a function $u \in C^{\infty}(M)$ proving that among our star products $\star_{\kappa}$ there are star products of (anti-)standard-ordered type. Finally, we find conditions on which the constructed star products are of Weyl type.

Proposition 4.1. Let $\kappa \in \mathbb{R}$.

i) The element $r_{\kappa} \in \mathcal{W}_{1} \otimes \mathcal{S} \otimes \Lambda^{1}$ constructed in Theorem 3.3 satisfies $\mathcal{H} r_{\kappa}=r_{\kappa}$ iff $\operatorname{deg}_{\lambda} B=$ $B$, which is the case iff $B=\lambda B_{1}$. In this case, $\mathcal{H}$ commutes with $\mathcal{D}_{\kappa}$ implying that $\mathcal{H} \tau_{\kappa}(s)=\tau_{\kappa}(\mathcal{H} s)$ for all $s \in \mathcal{S}$ which in turn shows that

$$
\mathcal{H}\left(s *_{\kappa} t\right)=(\mathcal{H} s) *_{\kappa} t+s *_{\kappa}(\mathcal{H} t)
$$

holds for all $s, t \in \mathcal{S}$.

ii) In case $B$ satisfies $\operatorname{deg}_{\lambda} B=B$ the star product $\star_{\kappa}$ on $C^{\infty}\left(E^{*}\right)[[\lambda]]$ obtained from the Fedosov construction is homogeneous, i.e.

$$
\mathrm{H}\left(f \star_{\kappa} g\right)=(\mathrm{H} f) \star_{\kappa} g+f \star_{\kappa}(\mathrm{H} g)
$$

for all $f, g \in C^{\infty}\left(E^{*}\right)[[\lambda]]$.

Proof. Part ii) is obvious from the observation that $\mathrm{H}=\mathcal{J} \circ \mathcal{H} \circ \mathcal{J}^{-1}$ and the very definition of $\star_{\kappa}$. One just has to observe that the identity (4.2) is an equation between (bi)differential operators which is satisfied in case it is satisfied on all of $\mathrm{Pol}^{\bullet}\left(E^{*}\right)$ which is the case according to part $i$ ). Thus we are left with the proof of the first part of the proposition. Applying $\mathcal{H}$ to the equations determining $r_{\kappa}$ and combining the resulting equations with the original ones one gets $\delta^{-1}\left(\mathcal{H} r_{\kappa}-r_{\kappa}\right)=0$ and $\delta\left(\mathcal{H} r_{\kappa}-r_{\kappa}\right)=D\left(\mathcal{H} r_{\kappa}-r_{\kappa}\right)+\frac{i}{\lambda} \operatorname{ad}_{\kappa}\left(r_{\kappa}\right)\left(\mathcal{H} r_{\kappa}-r_{\kappa}\right)+\operatorname{deg}_{\lambda} B-B$. Therefore $\mathcal{H} r_{\kappa}=r_{\kappa}$ evidently implies $\operatorname{deg}_{\lambda} B=B$. Vice versa supposing $\operatorname{deg}_{\lambda} B=B$ the above identities combine to $\mathcal{H} r_{\kappa}-r_{\kappa}=\delta^{-1}\left(D\left(\mathcal{H} r_{\kappa}-r_{\kappa}\right)+\frac{i}{\lambda} \operatorname{ad}_{\kappa}\left(r_{\kappa}\right)\left(\mathcal{H} r_{\kappa}-r_{\kappa}\right)\right)$ which is a fixed point equation with a unique solution that trivially is solved by zero and hence $\mathcal{H} r_{\kappa}=r_{\kappa}$. Using that $\mathcal{H}$ is a derivation of $\circ_{\kappa}$ it is straightforward to verify that in this case $\left[\mathcal{H}, \mathcal{D}_{\kappa}\right]=0$. Applying this identity to $\tau_{\kappa}(s)$ one finds $\mathcal{D}_{\kappa}\left(\mathcal{H} \tau_{\kappa}(s)\right)=0$ and therefore using Theorem 3.4 we have $\mathcal{H} \tau_{\kappa}(s)=\tau_{\kappa}\left(\sigma\left(\mathcal{H} \tau_{\kappa}(s)\right)\right)=\tau_{\kappa}(\mathcal{H} s)$, where the last equality follows from the fact that $\sigma$ commutes with $\mathcal{H}$. Finally, equation (4.1) is a direct consequence of the very definition of $*_{\kappa}$ and the identity for $\mathcal{H} \tau_{\kappa}(s)$ just shown.

Now we turn to the more detailed consideration of the recursion formulas for $r_{\kappa}$.

Proposition 4.2. Let $\kappa \in \mathbb{R}$.

i) For all $\mathrm{d}_{E}$-closed $B \in \Gamma^{\infty}\left(\Lambda^{2} E^{*}\right)[[\lambda]]$ the element $r_{\kappa} \in \mathcal{W}_{1} \otimes \mathcal{S} \otimes \Lambda^{1}$ is of $\mathrm{S}^{\bullet} E$-degree at most 1 and is determined by the simplified recursion formula

$$
r_{\kappa}=\delta^{-1}\left(D r_{\kappa}+\mathrm{i}_{\mathrm{s}}\left(e^{\alpha}\right) r_{\kappa} \mathrm{i}_{\mathrm{S}}\left(e_{\alpha}\right) r_{\kappa}-R+B\right) .
$$

In particular, this shows that $r_{\kappa}$ is actually independent of $\kappa$. Henceforth we thus can neglect the additional index $\kappa$ and write $r$ for $r_{\kappa}$. 
ii) For all $B$ as above $r$ can be written as $r=r_{0}+r_{1}$. Here $r_{1}$ denotes the solution of (3.4) for $B=0$, which is homogeneous of $\mathrm{S}^{\bullet}$ E-degree 1 and $\mathrm{r}_{0}$, which is homogeneous of $\mathrm{S}^{\bullet} \mathrm{E}$ degree 0 and explicitly given by

$$
\mathrm{r}_{0}=\frac{1}{\mathrm{id}-\delta^{-1}\left(D+\mathrm{i}_{\mathrm{s}}\left(e^{\alpha}\right) \mathrm{r}_{1} \mathrm{i}_{\mathrm{s}}\left(e_{\alpha}\right)\right)} \delta^{-1} B=\frac{1}{\mathrm{id}-\left[\delta^{-1}, D+\mathrm{i}_{\mathrm{s}}\left(e^{\alpha}\right) \mathrm{r}_{1} \mathrm{i}_{\mathrm{s}}\left(e_{\alpha}\right)\right]} \delta^{-1} B .
$$

In particular, $\mathrm{r}_{0}$ depends linearly on $B$.

Proof. We prove assertion $i$ ) by induction on the total degree. From the recursion formula (3.5) we find $r_{\kappa}^{(1)}=\delta^{-1} B_{0}, r_{\kappa}^{(2)}=\delta^{-1}\left(D r_{\kappa}^{(1)}+\frac{\mathrm{i}}{\lambda} r_{\kappa}^{(1)} \mathrm{o}_{\kappa} r_{\kappa}^{(1)}-R+\lambda B_{1}\right)$ and

$$
r_{\kappa}^{(k+1)}=\delta^{-1}\left(D r_{\kappa}^{(k)}+\frac{\mathrm{i}}{\lambda} \sum_{\ell=1}^{k} r_{\kappa}^{(\ell)} \circ_{\kappa} r_{\kappa}^{(k+1-\ell)}+\lambda^{k} B_{k}\right)
$$

for $k \geq 2$. Obviously, $r_{\kappa}^{(1)}$ is of $S^{\bullet} E$-degree 0 . Using this observation together with the explicit shape for $\circ_{\kappa}$ yields $r_{\kappa}^{(2)}=\delta^{-1}\left(D r_{\kappa}^{(1)}-R+\lambda B_{1}\right)$ which is easily seen to be of $S^{\bullet} E$-degree at most 1 . Now assume that we already have shown that $r_{\kappa}^{(j)}$ is of $S^{\bullet} E$-degree at most 1 for $j=1, \ldots, k$, then again use of the explicit shape of $o_{\kappa}$ yields by straightforward computation that

$$
r_{\kappa}^{(k+1)}=\delta^{-1}\left(D r_{\kappa}^{(k)}+\sum_{\ell=1}^{k} \mathrm{i}_{\mathrm{S}}\left(e^{\alpha}\right) r_{\kappa}^{(\ell)} \mathrm{i}_{\mathrm{S}}\left(e_{\alpha}\right) r_{\kappa}^{(k+1-\ell)}+\lambda^{k} B_{k}\right) .
$$

But this expression is obviously of $\mathrm{S}^{\bullet} E$-degree at most 1 in case $r_{\kappa}^{(1)}, \ldots, r_{\kappa}^{(k)}$ are. Recollecting the terms of each total degree into one element $r_{\kappa}$ finally shows the simplified recursion formula (4.3). According to $i$ ) we can write $r=\mathrm{r}_{0}+\mathrm{r}_{1}$, where $\mathrm{r}_{0}$ and $\mathrm{r}_{1}$ are homogeneous of $\mathrm{S} \bullet E$-degree 0 and 1. Inserting this decomposition into the above recursion formula yields considering the parts of degree 1 and 0 separately $\mathrm{r}_{1}=\delta^{-1}\left(D \mathrm{r}_{1}+\mathrm{i}_{\mathrm{s}}\left(e^{\alpha}\right) \mathrm{r}_{1} \mathrm{i}_{\mathrm{s}}\left(e_{\alpha}\right) \mathrm{r}_{1}-R\right)$, which coincides with the equation for $r$ in case $B=0$, and $\mathrm{r}_{0}=\delta^{-1}\left(D \mathrm{r}_{0}+\mathrm{i}_{\mathrm{s}}\left(e^{\alpha}\right) \mathrm{r}_{1} \mathrm{i}_{\mathrm{s}}\left(e_{\alpha}\right) \mathrm{r}_{0}+B\right)$. This equation is uniquely solved by (4.4).

Remark 4.3. One should note, that the homogeneity of $r_{1}$ according to Proposition 4.1, i.e. $\mathcal{H} r_{1}=r_{1}$ together with the fact that $\operatorname{deg}_{\text {ver }} r_{1}=r_{1}$ directly implies that $\operatorname{deg}_{\lambda} r_{1}=0$, i.e. $r_{1}$ is purely classical. Furthermore, the above proposition shows that $r$ decomposes into one classical part $r_{1}$ that is independent of $B$ and another non-classical part $r_{0}$ that completely encodes the dependence of $r$ on $B$.

To write down the explicit formulas for the $\star_{\kappa}$-product with a function $\pi^{*} u$ the following notions turn out to be very useful. By $\mathrm{D}: \Gamma^{\infty}\left(\mathrm{S}^{\bullet} E^{*}\right) \longrightarrow \Gamma^{\infty}\left(\mathrm{S}^{\bullet+1} E^{*}\right)$ we denote the operator of symmetric E-covariant derivation

$$
\mathrm{D}=e^{\alpha} \nabla_{e_{\alpha}} .
$$

For functions $u \in C^{\infty}(M)$ and one-forms $\alpha \in \Gamma^{\infty}\left(E^{*}\right)$ we define the algebraic differential operators $\mathcal{F}(u)$ and $\mathcal{F}(\alpha)$ on $\mathcal{S}^{\bullet}(E)$ by

$$
\mathcal{F}(u) t=u t \quad \text { and } \quad \mathcal{F}(\alpha) t=\mathrm{i}_{\mathrm{s}}(\alpha) t
$$

and extend the map $\mathcal{F}$ to a homomorphism from $\mathcal{S}^{\bullet}\left(E^{*}\right)=\bigoplus_{k=0}^{\infty} \Gamma^{\infty}\left(\mathrm{S}^{k} E^{*}\right)$ to the algebraic differential operators on $\mathcal{S}^{\bullet}(E)$. For obvious reasons we sometimes refer to the operators in the image of $\mathcal{F}$ as fibre derivatives, see [2] for a motivation. Analogously, we define $\mathrm{F}(u) f=\pi^{*} u f$ and $\mathrm{F}(\alpha) f=\mathscr{L}_{\alpha^{\text {ver }}} f$ for $f \in C^{\infty}\left(E^{*}\right)$ and extend $\mathrm{F}$ to a homomorphism from $\mathcal{S}^{\bullet}\left(E^{*}\right)$ to the differential operators on $C^{\infty}\left(E^{*}\right)$. Clearly, for all $\alpha \in \mathcal{S}^{\bullet}\left(E^{*}\right)$ we therefore have the relation $\mathcal{J}^{-1} \circ \mathrm{F}(\alpha) \circ \mathcal{J}=\mathcal{F}(\alpha)$. 
Proposition 4.4. For all choices of $\mathrm{d}_{E}$-closed $B \in \Gamma^{\infty}\left(\Lambda^{2} E^{*}\right)[[\lambda]]$ one has:

i) The star products $\star_{\text {Std }}$ resp. $\star_{\overline{\text { Std }}}$ are of standard-ordered type resp. anti-standard-ordered type in the sense that

$$
\pi^{*} u \star_{\mathrm{Std}} f=\pi^{*} u f \quad \text { and } \quad f \star_{\overline{\mathrm{Std}}} \pi^{*} u=f \pi^{*} u
$$

for all $u \in C^{\infty}(M)[[\lambda]]$ and all $f \in C^{\infty}\left(E^{*}\right)[[\lambda]]$. Equivalently, $\star_{\text {Std }}\left(\right.$ resp. $\left.\star_{\overline{\text { Std }}}\right)$ differentiates the first (resp. second) argument only in fibre directions.

ii) The Fedosov-Taylor series of $u \in C^{\infty}(M)[[\lambda]]$ is explicitly given by

$$
\tau_{\kappa}(u)=\exp (\mathrm{D}) u
$$

iii) In addition, the $\star_{\kappa}$-left and $\star_{\kappa}$-right multiplications with $\pi^{*} u$ can be expressed as

$$
\pi^{*} u \star_{\kappa} f=\mathrm{F}(\exp (\kappa \mathrm{i} \lambda \mathrm{D}) u) f \quad \text { and } \quad f \star_{\kappa} \pi^{*} u=\mathrm{F}(\exp (-(1-\kappa) \mathrm{i} \lambda \mathrm{D}) u) f .
$$

Consequently, for all $u \in C^{\infty}(M)[[\lambda]]$ one has

$$
\operatorname{ad}_{\star_{\kappa}}\left(\pi^{*} u\right)=\mathrm{F}\left(\frac{\exp (\kappa \mathrm{i} \lambda \mathrm{D})-\exp (-(1-\kappa) \mathrm{i} \lambda \mathrm{D})}{\mathrm{D}} \mathrm{d}_{E} u\right)
$$

iv) For all $\star_{\kappa}$ the functions $\pi^{*} C^{\infty}(M)[[\lambda]]$ are a subalgebra with $\star_{\kappa}$ being the undeformed commutative product.

Proof. Assertion $i)$ follows from $u *_{\text {Std }} t=\sigma\left(\tau_{\text {Std }}(u) \circ_{\text {Std }} \tau_{\text {Std }}(t)\right)=\sigma\left(\left(\sigma\left(\tau_{\text {Std }}(u)\right)\right) \circ_{\text {Std }} \tau_{\text {Std }}(t)\right)=$ $\sigma\left(u \circ_{\text {Std }} \tau_{\text {Std }}(t)\right)=\sigma\left(u \tau_{\text {Std }}(t)\right)=u t$, where the second equality is due to the explicit shape of $\circ_{\text {Std }}$. Observe that one does not need to know $\tau_{\mathrm{Std}}(u)$ in order to prove this statement. Analogously one shows $t * \overline{\operatorname{std}} u=t u$ for all $u \in C^{\infty}(M)[[\lambda]]$ and all $t \in \mathcal{S}$. From the very definitions of $\star_{\text {Std }}$ and $\star_{\text {Std }}$ we can therefore conclude that the equations (4.5) hold since they hold on $\operatorname{Pol}^{\bullet}\left(E^{*}\right)[[\lambda]]$. The second statement follows using associativity. For the proof of $\left.i i\right)$ we just have to show that $\exp (\mathrm{D}) u=u+\delta^{-1}\left(D \exp (\mathrm{D}) u+\frac{\mathrm{i}}{\lambda} \operatorname{ad}_{\kappa}(r) \exp (\mathrm{D}) u\right)$, which is straightforward observing that for all $a \in \mathcal{W}$ with $\operatorname{deg}_{\mathrm{s}} a=k a$ we have $\delta^{-1} D a=\frac{1}{k+1} \mathrm{D} a$ and $\delta^{-1} \frac{\mathrm{i}}{\lambda} \operatorname{ad}_{\kappa}(r) a=$ $\delta^{-1}\left(\mathrm{i}_{\mathrm{s}}\left(e^{\alpha}\right) r \mathrm{i}_{\mathrm{s}}\left(e_{\alpha}\right) a\right)=0$, where the second to last equality is a consequence of the explicit shape of $\circ_{\kappa}$ and Proposition $4.2 i$ ). Moreover, we have used that $\delta^{-1} r=0$ according to (3.4). The first statement in $i$ ii) follows using $i$ ) similarly to the computation in $i$ )

$$
\begin{aligned}
u *_{\kappa} t & =\sigma\left(\tau_{\kappa}(u) \circ_{\kappa} \tau_{\kappa}(t)\right) \\
& =\sum_{k=0}^{\infty} \frac{1}{k !}(\kappa \mathrm{i} \lambda)^{k} \mathrm{i}_{\mathrm{s}}\left(e_{\alpha_{1}}\right) \cdots \mathrm{i}_{\mathrm{S}}\left(e_{\alpha_{k}}\right) \frac{1}{k !} \mathrm{D}^{k} u \mathrm{i}_{\mathrm{s}}\left(e^{\alpha_{1}}\right) \cdots \mathrm{i}_{\mathrm{S}}\left(e^{\alpha_{k}}\right) t \\
& =\mathcal{F}(\exp (\kappa \mathrm{i} \lambda \mathrm{D}) u) t .
\end{aligned}
$$

From this equation and the relation between $F$ and $\mathcal{F}$ we conclude the first equation in (4.6). The proof of the second equation for the right-multiplication with $\pi^{*} u$ is completely analogous. The last assertion in iii) then follows from the combination of the equations for $\pi^{*} u \star_{\kappa} f$ and $f \star_{\kappa} \pi^{*} u$ observing $\mathrm{D} u=\mathrm{d}_{E} u$. Finally, $\left.i v\right)$ is a direct consequence of $i i i$ ).

We conclude this section noting that - in case $B$ has been chosen appropriately - the star products $\star_{\text {Weyl }}$ are in fact of Weyl type, i.e. the complex conjugation $\mathrm{C}$ as well as the $\lambda$-parity operator $\mathrm{P}=(-1)^{\operatorname{deg}_{\lambda}}$ are anti-automorphisms of $\star_{\text {Weyl }}$ (cf. [38]). Note that we consider the formal parameter $\lambda$ as real and hence define $\mathrm{C} \lambda=\lambda$. 
Proposition 4.5. For $\star_{\mathrm{Weyl}}=\star_{\frac{1}{2}}$ we have the following statements:

i) In case $\mathrm{C} B=B$ the star product $\star_{\text {Weyl }}$ is Hermitian, i.e. $\mathrm{C}\left(f \star_{\text {Weyl }} g\right)=(\mathrm{C} g) \star_{\text {Weyl }}(\mathrm{C} f)$ for all $f, g \in C^{\infty}\left(E^{*}\right)[[\lambda]]$.

ii) In case $\mathrm{P} B=B$ the star product $\star_{\text {Weyl }}$ has the $\lambda$-parity property, i.e. $\mathrm{P}\left(f \star_{\text {Weyl }} g\right)=$ $(\mathrm{P} g) \star_{\text {Weyl }}(\mathrm{P} f)$ for all $f, g \in C^{\infty}\left(E^{*}\right)[[\lambda]]$.

iii) In case $\mathrm{C} B=B=\mathrm{P} B$ the star product $\star_{\text {Weyl }}$ is of Weyl type.

Proof. For the proof of $i)$ we first note that $\mathrm{C}\left(a \circ_{\text {Weyl }} b\right)=(-1)^{k l}(\mathrm{C} b) \circ_{\text {Weyl }}(\mathrm{C} a)$ for all $a \in$ $\mathcal{W} \otimes \mathcal{S} \otimes \Lambda^{k}, b \in \mathcal{W} \otimes \mathcal{S} \otimes \Lambda^{l}$. With $\mathrm{C} B=B$ and this property it is straightforward to show that $\mathrm{C} r$ satisfies the same equations as $r$ and hence $\mathrm{Cr}=r$ by the uniqueness of the solution of (3.4). Therefore $\mathrm{C}$ commutes with $\mathcal{D}_{\text {weyl }}$ implying $\mathrm{C} \tau_{\text {Weyl }}(s)=\tau_{\text {weyl }}(\mathrm{C} s)$ for all $s \in \mathcal{S}$. From this equation and the behaviour of $\circ_{\text {Weyl }}$ with respect to complex conjugation the identity $\mathrm{C}\left(s *_{\text {Weyl }} t\right)=(\mathrm{C} t) *_{\text {Weyl }}(\mathrm{C} s)$ for all $s, t \in \mathcal{S}$ is obvious implying the assertion. The proof of $\left.i i\right)$ is completely analogous to that of $i$ ) replacing $\mathrm{C}$ by $\mathrm{P}$ and $i i i)$ is just the combination of $i$ ) and $i$ ).

\section{Equivalence transformations}

In this section we will explicitly construct isomorphisms or equivalence transformations, respectively, relating the star products obtained for different values $\kappa$ and $\kappa^{\prime}$ of the ordering parameter, different torsion-free $E$-connections $\nabla$ and $\nabla^{\prime}$, and from cohomologous $E$-two-forms $B$ and $B^{\prime}$.

We begin with the construction of isomorphisms between products $*_{\kappa}$ and $*_{\kappa}^{\prime}$ obtained using the same ordering parameter $\kappa$, the same $E$-connection $\nabla$ and different but cohomologous formal series $B$ and $B^{\prime}$ of $\mathrm{d}_{E}$-closed $E$-two-forms. We look for an automorphism $\mathcal{A}_{h}$ of $\left(\mathcal{W} \otimes \mathcal{S} \otimes \Lambda, \circ_{\kappa}\right)$ of the form

$$
\mathcal{A}_{h}=\exp \left(-\frac{\mathrm{i}}{\lambda} \operatorname{ad}_{\kappa}(h)\right) \quad \text { with } \quad h \in \mathcal{W}_{1} \quad \text { and } \quad \sigma(h)=0
$$

such that

$$
\mathcal{D}_{\kappa}^{\prime}=\mathcal{A}_{h} \mathcal{D}_{\kappa} \mathcal{A}_{-h}
$$

In case we can find such a fibrewise automorphism the map $\mathcal{J}_{h}: \mathcal{S} \longrightarrow \mathcal{S}$ which is defined by

$$
\mathcal{J}_{h}(s)=\sigma\left(\mathcal{A}_{h} \tau_{\kappa}(s)\right)
$$

turns out to be an isomorphism from $*_{\kappa}$ to $*_{\kappa}^{\prime}$ since the relation between the Fedosov derivations implies that $\mathcal{A}_{h} \tau_{\kappa}(s)=\tau_{\kappa}^{\prime}\left(\mathcal{J}_{h} s\right)$ holds for all $s \in \mathcal{S}$. Now by a direct computation one finds that $\mathcal{A}_{h} \mathcal{D}_{\kappa} \mathcal{A}_{-h}=\mathcal{D}_{\kappa}+\frac{\mathrm{i}}{\lambda} \operatorname{ad}_{\kappa}\left(\frac{\exp \left(-\frac{\mathrm{i}}{\lambda} \operatorname{ad}_{\kappa}(h)\right)-\mathrm{id}}{-\frac{\mathrm{i}}{\lambda} \operatorname{ad}_{\kappa}(h)} \mathcal{D}_{\kappa} h\right)$ which is equal to $\mathcal{D}_{\kappa}^{\prime}$ iff

$$
\frac{\mathrm{i}}{\lambda} \operatorname{ad}_{\kappa}\left(r-r^{\prime}+\frac{\exp \left(-\frac{\mathrm{i}}{\lambda} \operatorname{ad}_{\kappa}(h)\right)-\mathrm{id}}{-\frac{\mathrm{i}}{\lambda} \operatorname{ad}_{\kappa}(h)} \mathcal{D}_{\kappa} h\right)=0 .
$$

By Lemma 3.1, this is the case iff there is a formal series of $E$-one-forms $A$ such that

$$
r-r^{\prime}+\frac{\exp \left(-\frac{\mathrm{i}}{\lambda} \operatorname{ad}_{\kappa}(h)\right)-\mathrm{id}}{-\frac{\mathrm{i}}{\lambda} \operatorname{ad}_{\kappa}(h)} \mathcal{D}_{\kappa} h=-A .
$$


Now using that $h$ is of $S^{\bullet} E$-degree 0 together with Proposition $4.2 i$ ) it is easy to show that $\mathcal{D}_{\kappa} h$ is also of $\mathrm{S}^{\bullet} E$-degree 0 which implies that the above equation reduces to

$$
\mathcal{D}_{\kappa} h=r^{\prime}-r-A,
$$

since $\mathcal{W} \otimes \mathcal{S}^{0} \otimes \Lambda$ is a super-commutative subalgebra of $\left(\mathcal{W} \otimes \mathcal{S} \otimes \Lambda, \circ_{\kappa}\right)$. Applying $\mathcal{D}_{\kappa}$ to this equation, we get using the equations for $r$ and $r^{\prime}$ that the necessary condition for the solvability of this equation is that $A$ satisfies

$$
B-B^{\prime}=\mathrm{d}_{E} A .
$$

But this is also sufficient since the $\mathcal{D}_{\kappa}$-cohomology is trivial on elements with positive $\Lambda^{\bullet} E^{*}$ degree and the solution $h_{A}$ is explicitly given by $h_{A}=\mathcal{D}_{\kappa}^{-1}\left(r^{\prime}-r-A\right)$. We thus have shown the first part of the following:

Theorem 5.1. Let $*_{\kappa}$ and $*_{\kappa}^{\prime}$ be Fedosov products on $\mathcal{S}$ obtained using the same E-connection and different but cohomologous $\mathrm{d}_{E}$-closed $E$-two-forms $B$ and $B^{\prime}$.

i) In case $A$ satisfies $B-B^{\prime}=\mathrm{d}_{E} A$ there is a uniquely determined element $h_{A} \in \mathcal{W}_{1}$ with $\sigma\left(h_{A}\right)=0$ such that $\mathcal{D}_{\kappa} h_{A}=r^{\prime}-r-A$ namely

$$
h_{A}=\mathcal{D}_{\kappa}^{-1}\left(r^{\prime}-r-A\right) .
$$

With the so-constructed $h_{A}$ one has $\mathcal{D}_{\kappa}^{\prime}=\mathcal{A}_{h_{A}} \mathcal{D}_{\kappa} \mathcal{A}_{-h_{A}}$ and thus $\mathcal{J}_{A}=\mathcal{J}_{h_{A}}$ according to equation (5.2) defines an algebra isomorphism from $\left(\mathcal{S}, *_{\kappa}\right)$ to $\left(\mathcal{S}, *_{\kappa}^{\prime}\right)$.

ii) The above element $h_{A} \in \mathcal{W}_{1}$ is explicitly given by

$$
h_{A}=\frac{\exp (\mathrm{D})-\mathrm{id}}{\mathrm{D}} A
$$

where the formal series of E-one-forms $A$ is considered as a one-form in the symmetric part of $\mathcal{W} \otimes \mathcal{S} \otimes \Lambda$, i.e. as element of $\mathcal{W}^{1}$. Moreover, the isomorphism $\mathcal{J}_{A}$ takes the concrete form

$$
\mathcal{J}_{A} s=\exp \left(\mathcal{F}\left(\frac{\exp (\kappa \mathrm{i} \lambda \mathrm{D})-\exp (-(1-\kappa) \mathrm{i} \lambda \mathrm{D})}{\mathrm{i} \lambda \mathrm{D}} A\right)\right) s
$$

for $s \in \mathcal{S}$.

iii) The map $\mathrm{I}_{A}$ defined by

$$
\mathrm{I}_{A}=\Phi_{A_{0}}^{*} \circ \exp \left(\mathrm{F}\left(\frac{\exp (\kappa \mathrm{i} \lambda \mathrm{D})-\exp (-(1-\kappa) \mathrm{i} \lambda \mathrm{D})}{\mathrm{i} \lambda \mathrm{D}} A-A_{0}\right)\right)
$$

where $A_{0}$ denotes the classical part of $A$ and $\Phi_{A_{0}}: E^{*} \longrightarrow E^{*}$ denotes the fibre translating diffeomorphism defined by

$$
\Phi_{A_{0}}\left(\alpha_{q}\right)=\alpha_{q}+A_{0}(q)
$$

provides an isomorphism from $\left(C^{\infty}\left(E^{*}\right)[[\lambda]], \star_{\kappa}\right)$ to $\left(C^{\infty}\left(E^{*}\right)[[\lambda]], \star_{\kappa}^{\prime}\right)$.

iv) In case $\operatorname{deg}_{\lambda} B=B$ and $\operatorname{deg}_{\lambda} B^{\prime}=B^{\prime}$, we can choose $A$ such that $\operatorname{deg}_{\lambda} A=A$, too. Then $\mathrm{I}_{A}$ is homogeneous, i.e. $\mathrm{I}_{A} \mathrm{H}=\mathrm{HI}_{A}$. 
Proof. For the proof of the second part we have to evaluate $\mathcal{D}_{\kappa}^{-1}\left(r^{\prime}-r-A\right)$ using the concrete shape of the homotopy operator $\mathcal{D}_{\kappa}^{-1}$ explicitly. Since $\left[\delta^{-1},\left[\delta^{-1}, D+\frac{\mathrm{i}}{\lambda} \operatorname{ad}_{\kappa}(r)\right]\right]=0$ the terms involving $r^{\prime}-r$ vanish due to $\delta^{-1} r=0=\delta^{-1} r^{\prime}$ yielding $h_{A}=\frac{1}{\mathrm{id}-\left[\delta^{-1}, D+\frac{\mathrm{i}}{\lambda} \operatorname{ad}_{\kappa}(r)\right]} A$, where $A$ is viewed as element in $\mathcal{W}^{1}$. But as $\left[\delta^{-1}, D+\frac{\mathrm{i}}{\lambda} \operatorname{ad}_{\kappa}(r)\right]$ preserves $\mathcal{W}$ and $\left[\delta^{-1}, \frac{\mathrm{i}}{\lambda} \operatorname{ad}_{\kappa}(r)\right]$ even vanishes on $\mathcal{W}$ we get $h_{A}=\frac{1}{\mathrm{id}-\left[\delta^{-1}, D\right]} A=\frac{\exp (\mathrm{D}) \text {-id }}{\mathrm{D}} A$ where in the last step we have used that $\left[\delta^{-1}, D\right] a=\frac{1}{k+1} \mathrm{D} a$ for all $a \in \mathcal{W}$ with $\operatorname{deg}_{\mathrm{s}} a=k a$ and have explicitly computed the geometric series. For the detailed computation of $\mathcal{J}_{A} s$ we first note that $\sigma\left(\operatorname{ad}_{\kappa}(a) b\right)=\sigma\left(\operatorname{ad}_{\kappa}(a) \sigma(b)\right)$ for all $a \in \mathcal{W} \otimes \mathcal{S}^{0} \otimes \Lambda$ and all $b \in \mathcal{W} \otimes \mathcal{S} \otimes \Lambda$ because of the explicit shape of $\circ_{\kappa}$. Repeated use of this identity yields $\mathcal{J}_{A} s=\exp \left(-\frac{\mathrm{i}}{\lambda} \sigma \circ \operatorname{ad}_{\kappa}\left(\frac{\exp (\mathrm{D})-\mathrm{id}}{\mathrm{D}} A\right)\right) s$. A further straightforward computation shows that

$$
\sigma\left(\operatorname{ad}_{\kappa}\left(\frac{\exp (\mathrm{D})-\mathrm{id}}{\mathrm{D}} A\right) s\right)=\mathcal{F}\left(\frac{\exp (\kappa \mathrm{i} \lambda \mathrm{D})-\exp (-(1-\kappa) \mathrm{i} \lambda \mathrm{D})}{\mathrm{D}} A\right) s
$$

for all $s \in \mathcal{S}$ proving the formula for $\mathcal{J}_{A}$. Evidently, from the very definition of $\star_{\kappa}$ and $\star_{\kappa}^{\prime}$ the map $\mathrm{I}_{A}=\mathcal{J} \circ \mathcal{J}_{A} \circ \mathcal{J}^{-1}$ defines an isomorphism from $\left(\mathrm{Pol}^{\bullet}\left(E^{*}\right)[[\lambda]], \star_{\kappa}\right)$ to $\left(\mathrm{Pol}^{\bullet}\left(E^{*}\right)[[\lambda]], \star_{\kappa}^{\prime}\right)$. But as $A$ starts in order 0 of the formal parameter the so-obtained map cannot be continued to a welldefined map on all of $C^{\infty}\left(E^{*}\right)[[\lambda]]$ without being suitably rewritten. This trouble is caused by the term $\exp \left(\mathcal{F}\left(A_{0}\right)\right)$ which is perfectly well defined on $\mathrm{Pol}^{\bullet}\left(E^{*}\right)$ but not on $C^{\infty}\left(E^{*}\right)$. But on $\mathrm{Pol}^{\bullet}\left(E^{*}\right)$ the map $\exp \left(\mathcal{F}\left(A_{0}\right)\right)$ coincides with the pull-back $\Phi_{A_{0}}^{*}$ with the above fibre translating diffeomorphism. Therefore on $\mathrm{Pol}^{\bullet}\left(E^{*}\right)[[\lambda]]$ the expression in equation (5.4) coincides with $\mathcal{J} \circ J_{A} \circ \mathcal{J}^{-1}$ but it is moreover well defined on all of $C^{\infty}\left(E^{*}\right)[[\lambda]]$. With the usual argument, that it suffices to verify identities relating the star products $\star_{\kappa}$ and $\star_{\kappa}^{\prime}$ on polynomial functions in order to prove them for all smooth functions, this implies that $\mathrm{I}_{A}$ defines an isomorphism from $\left(C^{\infty}\left(E^{*}\right)[[\lambda]], \star_{\kappa}\right)$ to $\left(C^{\infty}\left(E^{*}\right)[[\lambda]], \star_{\kappa}^{\prime}\right)$. The last part is obvious.

As a by-product of the above considerations and the formulas proven in Proposition 4.4 we obtain a unique characterization of the (quasi-inner) derivations and (inner) self-equivalences of $\star_{\kappa}$ that are in the image of $F$.

Corollary 5.2. The map

$$
A \mapsto \mathrm{F}\left(\frac{\exp (\kappa \mathrm{i} \lambda \mathrm{D})-\exp (-(1-\kappa) \mathrm{i} \lambda \mathrm{D})}{\mathrm{i} \lambda \mathrm{D}} A\right)
$$

establishes a bijection between $\left\{A \in \Gamma^{\infty}\left(E^{*}\right)[[\lambda]] \mid \mathrm{d}_{E} A=0\right\}$ and $\operatorname{Der}\left(C^{\infty}\left(E^{*}\right)[[\lambda]], \star_{\kappa}\right) \cap \operatorname{im}(\mathrm{F})$. Since all self-equivalences of $\star_{\kappa}$ are of the form $\exp (\mathrm{i} \lambda \mathrm{D})$ with $\mathrm{D} \in \operatorname{Der}\left(C^{\infty}\left(E^{*}\right)[[\lambda]], \star_{\kappa}\right)$ this also yields that all self-equivalences of $\star_{\kappa}$ that lie in $\operatorname{im}(\mathrm{F})$ have the form

$$
\exp \left(\mathrm{F}\left(\frac{\exp (\kappa \mathrm{i} \lambda \mathrm{D})-\exp (-(1-\kappa) \mathrm{i} \lambda \mathrm{D})}{\mathrm{D}} A\right)\right) .
$$

Moreover, the derivations obtained from $A=\mathrm{d}_{E} u$ with $u \in C^{\infty}(M)[[\lambda]]$ are of the form $-\frac{\mathrm{i}}{\lambda} \operatorname{ad}_{\star_{\kappa}}\left(\pi^{*} u\right)$ and hence are quasi-inner. Analogously, the self-equivalences corresponding to $A=\mathrm{d}_{E} u$ are given by $\exp \left(\operatorname{ad}_{\star_{\kappa}}\left(\pi^{*} u\right)\right) f=\mathrm{e}^{\pi^{*} u} \star_{\kappa} f \star_{\kappa} \mathrm{e}^{-\pi^{*} u}$ and hence are inner automorphisms.

Let us now construct an equivalence transformation from $\star_{\kappa}$ to $\star_{\kappa}^{\prime}$ obtained from different torsion-free $E$-connections $\nabla$ and $\nabla^{\prime}$ but coinciding formal series of $\mathrm{d}_{E}$-closed $E$-forms $B$. We begin with the comparison of the corresponding maps $D$ and $D^{\prime}$ on $\mathcal{W} \otimes \mathcal{S} \otimes \Lambda$. Evidently, $S_{\nabla-\nabla^{\prime}}(s, t)=\nabla_{s} t-\nabla_{s}^{\prime} t$ defines an element of $\Gamma^{\infty}\left(\mathrm{S}^{2} E^{*} \otimes E\right)$ which we naturally can consider as $S_{\nabla-\nabla^{\prime}} \in \mathcal{W}^{2} \otimes \mathcal{S}^{1}$. For $T_{\nabla-\nabla^{\prime}}=\delta S_{\nabla-\nabla^{\prime}} \in \mathcal{W}^{1} \otimes \mathcal{S}^{1} \otimes \Lambda^{1}$ we find: 
Lemma 5.3. With the definitions from above the following identities hold:

i)

$$
D-D^{\prime}=-\frac{\mathrm{i}}{\lambda} \operatorname{ad}_{\kappa}\left(T_{\nabla-\nabla^{\prime}}\right)
$$

ii)

$$
\delta T_{\nabla-\nabla^{\prime}}=0 \quad \text { and } \quad D T_{\nabla-\nabla^{\prime}}=R-R^{\prime}-\frac{\mathrm{i}}{\lambda} T_{\nabla-\nabla^{\prime} \circ_{\kappa}} T_{\nabla-\nabla^{\prime}}
$$

Proof. Part $i$ ) follows from an easy computation using the definition of $T_{\nabla-\nabla^{\prime}}$ and $\circ_{\kappa}$. The first statement in $i$ ) is trivial since $\delta^{2}=0$ and the first formula involving $R$ and $R^{\prime}$ follows from squaring the identity $D^{\prime}=D+\frac{\mathrm{i}}{\lambda} \operatorname{ad}_{\kappa}\left(T_{\nabla-\nabla^{\prime}}\right)$ and using the definitions of $R$ and $R^{\prime}$.

To construct an equivalence from $*_{\kappa}$ to $*_{\kappa}^{\prime}$ we again try to find a fibrewise automorphism $\mathcal{A}_{h}$ as in (5.1) such that $\mathcal{D}_{\kappa}^{\prime}=\mathcal{A}_{h} \mathcal{D}_{\kappa} \mathcal{A}_{-h}$ with $\sigma(h)=0$. As above this equation turns out to be equivalent to

$$
T_{\nabla-\nabla^{\prime}}+r^{\prime}-r-\frac{\exp \left(-\frac{\mathrm{i}}{\lambda} \operatorname{ad}_{\kappa}(h)\right)-\mathrm{id}}{-\frac{\mathrm{i}}{\lambda} \operatorname{ad}_{\kappa}(h)} \mathcal{D}_{\kappa} h
$$

being a central element in $\left(\mathcal{W} \otimes \mathcal{S} \otimes \Lambda, \circ_{\kappa}\right)$. The following proposition states the existence of such an element and hence yields the desired equivalence from $\star_{\kappa}$ to $\star_{\kappa}^{\prime}$.

\section{Proposition 5.4.}

i) There is a uniquely determined element $h_{\nabla-\nabla^{\prime}} \in \mathcal{W}_{2} \otimes \mathcal{S}$ with $\sigma\left(h_{\nabla-\nabla^{\prime}}\right)=0$ such that

$$
T_{\nabla-\nabla^{\prime}}+r^{\prime}-r-\frac{\exp \left(-\frac{\mathrm{i}}{\lambda} \operatorname{ad}_{\kappa}\left(h_{\nabla-\nabla^{\prime}}\right)\right)-\mathrm{id}}{-\frac{\mathrm{i}}{\lambda} \operatorname{ad}_{\kappa}\left(h_{\nabla-\nabla^{\prime}}\right)} \mathcal{D}_{\kappa} h_{\nabla-\nabla^{\prime}}=0,
$$

which can be determined recursively from

$$
\begin{aligned}
h_{\nabla-\nabla^{\prime}}= & \delta^{-1}\left(D h_{\nabla-\nabla^{\prime}}+\frac{\mathrm{i}}{\lambda} \operatorname{ad}_{\kappa}(r) h_{\nabla-\nabla^{\prime}}\right. \\
& \left.-\frac{-\frac{\mathrm{i}}{\lambda} \operatorname{ad}_{\kappa}\left(h_{\nabla-\nabla^{\prime}}\right)}{\exp \left(-\frac{\mathrm{i}}{\lambda} \operatorname{ad}_{\kappa}\left(h_{\nabla-\nabla^{\prime}}\right)\right)-\mathrm{id}}\left(T_{\nabla-\nabla^{\prime}}+r^{\prime}-r\right)\right) .
\end{aligned}
$$

With the so-determined $h_{\nabla-\nabla^{\prime}}$ the map $\mathcal{E}_{\nabla-\nabla^{\prime}}$ defined by

$$
\mathcal{E}_{\nabla-\nabla^{\prime}} s=\sigma\left(\mathcal{A}_{h_{\nabla-\nabla^{\prime}}} \tau_{\kappa}(s)\right)
$$

is an equivalence transformation from $\left(\mathcal{S}, *_{\kappa}\right)$ to $\left(\mathcal{S}, *_{\kappa}^{\prime}\right)$.

ii) Moreover, $h_{\nabla-\nabla^{\prime}}$ is of $\mathrm{S}^{\bullet} E$-degree at most 1 and hence it satisfies the simpler recursion formula

$$
\begin{aligned}
h_{\nabla-\nabla^{\prime}}= & \delta^{-1}\left(D h_{\nabla-\nabla^{\prime}}-\left\{r, h_{\nabla-\nabla^{\prime}}\right\}_{\mathrm{fib}}\right. \\
& \left.-\frac{\left\{h_{\nabla-\nabla^{\prime}}, \cdot\right\}_{\mathrm{fib}}}{\exp \left(\left\{h_{\nabla-\nabla^{\prime}}, \cdot\right\}_{\mathrm{fib}}\right)-\mathrm{id}}\left(T_{\nabla-\nabla^{\prime}}+r^{\prime}-r\right)\right) .
\end{aligned}
$$


iii) $\mathcal{E}_{\nabla-\nabla^{\prime}}$ induces an equivalence transformation from $\left(C^{\infty}\left(E^{*}\right)[[\lambda]], \star_{\kappa}\right)$ to $\left(C^{\infty}\left(E^{*}\right)[[\lambda]], \star_{\kappa}^{\prime}\right)$ by the unique extension from $\operatorname{Pol}^{\bullet}\left(E^{*}\right)[[\lambda]]$ to $C^{\infty}\left(E^{*}\right)[[\lambda]]$ of the equivalence transformation $\mathrm{E}_{\nabla-\nabla^{\prime}}=\mathcal{J} \circ \mathcal{E}_{\nabla-\nabla^{\prime}} \circ \mathcal{J}^{-1}:\left(\mathrm{Pol}^{\bullet}\left(E^{*}\right)[[\lambda]], \star_{\kappa}\right) \longrightarrow\left(\mathrm{Pol}^{\bullet}\left(E^{*}\right)[[\lambda]], \star_{\kappa}^{\prime}\right)$.

iv) In case $\operatorname{deg}_{\lambda} B=B$ we have $\mathcal{H} h_{\nabla-\nabla^{\prime}}=h_{\nabla-\nabla^{\prime}}$ and hence $\mathrm{E}_{\nabla-\nabla^{\prime}}$ is homogeneous.

Proof. Solving equation (5.5) for $\mathcal{D}_{\kappa} h$ and applying $\mathcal{D}_{\kappa}$ one obtains by a straightforward but cumbersome computation that the necessary condition for the solvability of this equation is satisfied. But this also turns out to be sufficient since (3.2) applied to $h$ yields the recursion formula for $h_{\nabla-\nabla^{\prime}}$ which has a unique solution by the usual fixed point argument. For the soconstructed $h_{\nabla-\nabla^{\prime}}$ it is evident that $\mathcal{E}_{\nabla-\nabla^{\prime}}$ is an isomorphism as stated. Using Proposition 3.6 and the fact that $h_{\nabla-\nabla^{\prime}} \in \mathcal{W}_{2} \otimes \mathcal{S}$ it is lenghty but not difficult to show that $\mathcal{E}_{\nabla-\nabla^{\prime}}$ is in fact a formal series of differential operators that starts with the identity. The proof of $i i$ ) is a straightforward induction on the total degree using Proposition $4.2 i$ ), the shape of $T_{\nabla-\nabla^{\prime}}$, and the fact that the ${ }_{\kappa}$-super-commutator of two elements that are of $S^{\bullet} E$-degree at most 1 is of $\mathrm{S}^{\bullet} E$-degree at most 1 , too, and equals $i \lambda$ times the fibrewise Poisson bracket $\{\cdot, \cdot\}_{\text {fib }}$. Assertion iii) is a direct consequence of the fact that $\mathcal{E}_{\nabla-\nabla^{\prime}}$ is a formal series of differential operators which is an equivalence between differential associative products on $\mathcal{S}$. The last part follows from Proposition $4.1 \mathrm{i}$ ) together with (5.6) since $\mathcal{H}\left(T_{\nabla-\nabla^{\prime}}=T_{\nabla-\nabla^{\prime}}\right.$.

Finally, we can now compare the star products $\star_{\kappa}$ and $\star_{\kappa^{\prime}}$ obtained from different ordering parameters $\kappa$ and $\kappa^{\prime}$ but identical $\nabla$ and $B$. Actually, here the procedure is a little more involved since we do not only need a fibrewise automorphism but also the fibrewise equivalence transformation $\mathcal{M}_{\kappa^{\prime}-\kappa}$ from $\circ_{\kappa}$ to $\circ_{\kappa^{\prime}}$ defined in (3.3). We consider $\hat{\mathcal{D}}_{\kappa^{\prime}}=\mathcal{M}_{\kappa^{\prime}-\kappa} \mathcal{D}_{\kappa} \mathcal{M}_{\kappa-\kappa^{\prime}}$ which is evidently a super-derivation of $\circ_{\kappa^{\prime}}$ with square 0. Using Proposition $4.2 i$ ) and the commutation relations for $\Delta_{\text {fib }}$ we find

$$
\hat{\mathcal{D}}_{\kappa^{\prime}}=\mathcal{D}_{\kappa^{\prime}}+\left(\kappa-\kappa^{\prime}\right) \operatorname{ad}_{\kappa^{\prime}}\left(\Delta_{\text {fib }} r\right)
$$

which in general is evidently different from $\mathcal{D}_{\kappa^{\prime}}$. Therefore, we try to find $\mathcal{A}_{h}^{\prime}=\exp \left(-\frac{\mathrm{i}}{\lambda} \operatorname{ad}_{\kappa^{\prime}}(h)\right)$ with $h \in \mathcal{W}_{2}$ and $\sigma(h)=0$ such that $\mathcal{A}_{h}^{\prime} \hat{\mathcal{D}}_{\kappa^{\prime}} \mathcal{A}_{-h}^{\prime}=\mathcal{D}_{\kappa^{\prime}}$ since then analogously to the considerations before we can explicitly define an equivalence transformation from $\left(\mathcal{S}, *_{\kappa}\right)$ to $\left(\mathcal{S}, *_{\kappa^{\prime}}\right)$.

\section{Proposition 5.5.}

i) For every E-one-form $\Gamma$ with $\mathrm{d}_{E} \Gamma=-\Delta_{\text {fib }} R$ and all $\kappa, \kappa^{\prime} \in \mathbb{R}$ there is a uniquely determined element $h_{\Gamma, \kappa^{\prime}-\kappa} \in \mathcal{W}_{2}$ with $\sigma\left(h_{\Gamma, \kappa^{\prime}-\kappa}\right)=0$ such that

$$
\mathcal{D}_{\kappa^{\prime}} h_{\Gamma, \kappa^{\prime}-\kappa}=-\mathrm{i} \lambda\left(\kappa^{\prime}-\kappa\right)\left(\Gamma+\Delta_{\mathrm{fib}} r\right)
$$

namely $h_{\Gamma, \kappa^{\prime}-\kappa}=-\mathcal{D}_{\kappa^{\prime}}^{-1}\left(\mathrm{i} \lambda\left(\kappa^{\prime}-\kappa\right)\left(\Gamma+\Delta_{\text {fib }} r\right)\right)$.

ii) With the element $h_{\Gamma, \kappa^{\prime}-\kappa}$ constructed in i) one has $\mathcal{D}_{\kappa^{\prime}}=\mathcal{A}_{h_{\Gamma, \kappa^{\prime}-\kappa}^{\prime}}^{\prime} \mathcal{M}_{\kappa^{\prime}-\kappa} \mathcal{D}_{\kappa} \mathcal{M}_{\kappa-\kappa^{\prime}} \mathcal{A}_{h_{\Gamma, \kappa^{\prime}-\kappa}}^{\prime}$ and hence

$$
\mathcal{N}_{\Gamma, \kappa^{\prime}-\kappa} s=\sigma\left(\mathcal{A}_{h_{\Gamma, \kappa^{\prime}-\kappa}^{\prime}}^{\prime} \mathcal{M}_{\kappa^{\prime}-\kappa} \tau_{\kappa}(s)\right)
$$

defines an equivalence transformation from $\left(\mathcal{S}, *_{\kappa}\right)$ to $\left(\mathcal{S}, *_{\kappa^{\prime}}\right)$.

iii) Finally, the map $\mathrm{N}_{\Gamma, \kappa^{\prime}-\kappa}=\mathcal{J} \circ \mathcal{N}_{\Gamma, \kappa^{\prime}-\kappa} \circ \mathcal{J}^{-1}$ defines an equivalence transformation from $\left(\mathrm{Pol}^{\bullet}\left(E^{*}\right)[[\lambda]], \star_{\kappa}\right)$ to $\left(\mathrm{Pol}^{\bullet}\left(E^{*}\right)[[\lambda]], \star_{\kappa^{\prime}}\right)$ that uniquely extends to an equivalence transformation from $\left(C^{\infty}\left(E^{*}\right)[[\lambda]], \star_{\kappa}\right)$ to $\left(C^{\infty}\left(E^{*}\right)[[\lambda]], \star_{\kappa^{\prime}}\right)$.

iv) In case $\operatorname{deg}_{\lambda} B=B$, the equivalence transformation $\mathrm{N}_{\Gamma, \kappa^{\prime}-\kappa}$ is homogeneous. 
Proof. Using the shape of $\mathcal{A}_{h}^{\prime}$ which is a fibrewise automorphism of $\circ_{\kappa^{\prime}}$ we find that $\mathcal{A}_{h}^{\prime} \hat{\mathcal{D}}_{\kappa^{\prime}} \mathcal{A}_{-h}^{\prime}$ $=\mathcal{D}_{\kappa^{\prime}}$ iff

$$
\frac{\exp \left(-\frac{\mathrm{i}}{\lambda} \operatorname{ad}_{\kappa^{\prime}}(h)\right)-\mathrm{id}}{-\frac{\mathrm{i}}{\lambda} \operatorname{ad}_{\kappa^{\prime}}(h)} \mathcal{D}_{\kappa^{\prime}} h+\mathrm{i} \lambda\left(\kappa^{\prime}-\kappa\right) \mathcal{A}_{h}^{\prime} \Delta_{\mathrm{fib}} r
$$

is central. But now $\mathcal{D}_{\kappa^{\prime}} h \in \mathcal{W}_{2} \otimes \mathcal{S}^{0} \otimes \Lambda^{1}$ and $\Delta_{\text {fib }} r \in \mathcal{W} \otimes \mathcal{S}^{0} \otimes \Lambda^{1}$ together with the fact that $\mathcal{W} \otimes \mathcal{S}^{0} \otimes \Lambda$ is a super-commutative subalgebra of $\left(\mathcal{W} \otimes \mathcal{S} \otimes \Lambda, \circ_{\kappa^{\prime}}\right)$ imply that this is equivalent to

$$
\mathcal{D}_{\kappa^{\prime}} h+\mathrm{i} \lambda\left(\kappa^{\prime}-\kappa\right) \Delta_{\mathrm{fib}} r=A,
$$

with a formal series of $E$-one-forms $A$. Clearly, the necessary condition for the solvability of the latter equation is $\mathcal{D}_{\kappa^{\prime}}\left(\mathrm{i} \lambda\left(\kappa^{\prime}-\kappa\right) \Delta_{\text {fib }} r\right)=\mathrm{d}_{E} A$. In order to analyze this equation one applies $\mathcal{M}_{\kappa^{\prime}-\kappa}$ to the equation solved by $r$ with the fibrewise product $\circ_{\kappa}$ and finds $\mathcal{D}_{\kappa^{\prime}}\left(\mathrm{i} \lambda\left(\kappa^{\prime}-\kappa\right) \Delta_{\text {fib }} r\right)=$ $\mathrm{i} \lambda\left(\kappa^{\prime}-\kappa\right) \Delta_{\text {fib }} R$. Note that here the fact that $r$ does not depend on $\kappa$ proven in Proposition 4.2 enters crucially. But since there is an $E$-one-form $\Gamma$ with $\mathrm{d}_{E} \Gamma=-\Delta_{\text {fib }} R$ we can satisfy the above condition choosing $A=-\mathrm{i} \lambda\left(\kappa^{\prime}-\kappa\right) \Gamma$. Now again the homotopy operator $\mathcal{D}_{\kappa^{\prime}}^{-1}$ permits to write down the unique solution $h_{\Gamma, \kappa^{\prime}-\kappa}$ of (5.7). The assertion of $\left.i i\right)$ is then satisfied by construction of $h_{\Gamma, \kappa^{\prime}-\kappa}$ and part iii) follows again by the argument that $\mathcal{N}_{\Gamma, \kappa^{\prime}-\kappa}$ is a formal series of differential operators starting with the identity. For the last part, note that $\Delta_{\mathrm{fib}} r=\Delta_{\mathrm{fib}} \mathrm{r}_{1}$ is classical whence the right-hand side of (5.7) is homogeneous of degree one with respect to $\mathcal{H}$. This implies that $h_{\Gamma, \kappa^{\prime}-\kappa}$ is homogeneous of degree one, too. Since $\mathcal{M}_{\kappa^{\prime}-\kappa}$ commutes with $\mathcal{H}$, the result follows.

Remark 5.6. From the above statements it follows that the only possible source for non-equivalent star products in our framework are non-trivial cohomology classes $\left[B-B^{\prime}\right] \in \mathrm{H}_{E}^{2}(M)[[\lambda]]$. Note however, that even for $[B] \neq\left[B^{\prime}\right]$ the resulting star products can be equivalent: let $E=\mathfrak{g}$ be an Abelian even-dimensional Lie algebra, viewed as Lie algebroid over $\{\mathrm{pt}\}$. Choose $B_{0}$ to be a non-degenerate element in $\Lambda^{2} \mathfrak{g}^{*}$, whence the Poisson bracket $\{\cdot, \cdot\}_{B_{0}}$ is symplectic. Then any $B=B_{0}+\lambda B_{1}$ and $B^{\prime}=B_{0}+\lambda B_{1}^{\prime}$ are $\mathrm{d}_{E^{-}}$-closed and cohomologous only if $B_{1}=B_{1}^{\prime}$. Choosing $B_{1} \neq B_{1}^{\prime}$, the star products are still equivalent, as all symplectic star products on a vector space are equivalent.

\section{Relation to the universal enveloping algebra}

Now we relate the deformed algebra of polynomial functions $\left(\mathrm{Pol}^{\bullet}\left(E^{*}\right)[\lambda], \star_{\kappa}\right)$ for $B=0$, viewed as $\mathbb{C}[\lambda]$-subalgebra thanks to Proposition 1.2, to the universal enveloping algebra $\mathcal{U}(E)$ of the Lie algebroid $E$. First we recall the definition of $\mathcal{U}(E)$ according to Rinehart [42, Section 2]: The $\mathbb{C}[\lambda]$-module $\left(C^{\infty}(M) \oplus \Gamma^{\infty}(E)\right)[\lambda]$ is a Lie algebra over $\mathbb{C}[\lambda]$ via

$$
[(u, s),(v, t)]=-\mathrm{i} \lambda\left(\varrho(s) v-\varrho(t) u,[s, t]_{E}\right) .
$$

Note that we have incorporated artificially the pre-factor $\mathrm{i} \lambda$ which will allow for easier comparison. In its (Lie algebraic) universal enveloping algebra $\mathfrak{U}\left(\left(C^{\infty}(M) \oplus \Gamma^{\infty}(E)\right)[\lambda]\right.$ ) over $\mathbb{C}[\lambda]$ one considers the two-sided ideal $\mathfrak{I}$ generated by the relations $(u, 0) \diamond(0, s)-(0, u s)$ and $(u, 0) \diamond(v, 0)-(u v, 0)$, where $\diamond$ denotes the product of the universal enveloping algebra and $u, v \in C^{\infty}(M)[\lambda]$ and $s \in \Gamma^{\infty}(E)[\lambda]$. Then one defines

$$
\mathcal{U}(E)=\mathfrak{U}\left(\left(C^{\infty}(M) \oplus \Gamma^{\infty}(E)\right)[\lambda]\right) / \mathfrak{I} .
$$

The universal enveloping algebra $\mathcal{U}(E)$ (now in the Lie algebroid sense) is still filtered by the number of factors from $\Gamma^{\infty}(E)$. We denote by $\mathfrak{G r}^{\bullet}(\mathcal{U}(E))$ the corresponding graded $\mathbb{C}[\lambda]$-module, 
i.e. $\mathfrak{G r}^{k}(\mathcal{U}(E))=\mathcal{U}^{k}(E) / \mathcal{U}^{k-1}(E)$, endowed with its canonical algebra structure. It turns out that $\mathfrak{G r}^{\bullet}(\mathcal{U}(E))$ is a commutative unital $\mathbb{C}[\lambda]$-algebra, which is immediate from the particular form of the relations used in the definition of $\mathcal{U}(E)$. This algebra is still generated by $C^{\infty}(M)[\lambda]$ and $\Gamma^{\infty}(E)[\lambda]$ and $C^{\infty}(M)[\lambda] \longrightarrow \mathfrak{G r}^{0}(\mathcal{U}(E))$ turns out to be an algebra morphism. Thus $\mathfrak{G r}^{\bullet}(\mathcal{U}(E))$ is a $C^{\infty}(M)[\lambda]$-algebra, generated by $\Gamma^{\infty}(E)[\lambda]$. Hence we have a canonical algebra morphism

$$
\mathrm{S}_{C^{\infty}(M)[\lambda]}^{\bullet}\left(\Gamma^{\infty}(E)[\lambda]\right)=\mathcal{S}^{\bullet}(E)[\lambda] \longrightarrow \mathfrak{G r}^{\bullet}(\mathcal{U}(E))
$$

by the universal property of the symmetric algebra. Since $\Gamma^{\infty}(E)[\lambda]$ is a projective $C^{\infty}(M)[\lambda]$ module, which follows directly from the Serre-Swan theorem, it follows from [42, Theorem 3.1] that (6.1) is in fact an isomorphism, i.e. one has a Poincaré-Birkhoff-Witt like theorem. In particular, we can identify $u \in C^{\infty}(M)[\lambda]$ and $s \in \Gamma^{\infty}(E)[\lambda]$ with their images in $\mathcal{U}(E)$.

Now consider $\mathcal{S}^{\bullet}(E)[\lambda]$ (or equivalently, $\mathrm{Pol}^{\bullet}\left(E^{*}\right)[\lambda]$ ) with the (star) product $*_{\text {Weyl }}\left(\right.$ or $\star_{\text {Weyl }}$ ), where we have to choose $B=0$.

Proposition 6.1. For $B=0$ we have

$$
[u, v]_{*_{\mathrm{Weyl}}}=0, \quad[u, s]_{*_{\mathrm{Weyl}}}=\mathrm{i} \lambda \varrho(s) u, \quad \text { and } \quad[s, t]_{*_{\mathrm{Weyl}}}=-\mathrm{i} \lambda[s, t]_{E}
$$

for $u, v \in C^{\infty}(M)[\lambda]$ and $s, t \in \Gamma^{\infty}(E)[\lambda]$.

Proof. The first two statements are true by homogeneity, the last follows from homogeneity and the Weyl type property.

Remark 6.2. This proposition, transferred to $\mathrm{Pol}^{\bullet}\left(E^{*}\right)$, can be viewed as a covariance property of $\star_{\text {Weyl }}$ under the Lie algebra action of $C^{\infty}(M) \rtimes \Gamma^{\infty}(E)$ acting on $C^{\infty}\left(E^{*}\right)$ via inner Poisson derivations. Thus the map $\mathcal{J}$, restricted to $C^{\infty}(M) \rtimes \Gamma^{\infty}(E)$, can be considered as a quantum momentum map.

Lemma 6.3. There exists a unique surjective unital $\mathbb{C}[\lambda]$-linear algebra morphism

$$
\phi: \mathcal{U}(E) \longrightarrow\left(\mathcal{S}^{\bullet}(E)[\lambda], *_{\text {weyl }}\right)
$$

with $\phi(u)=u$ and $\phi(s)=s$ for $u \in C^{\infty}(M)[\lambda]$ and $s \in \Gamma^{\infty}(E)[\lambda]$.

Proof. By (6.2) and the universal property of $\mathcal{U}(E)$ existence and uniqueness of such a morphism are clear. The surjectivity follows since $\mathcal{S}^{\bullet}(E)[\lambda]$ is generated by $C^{\infty}(M)[\lambda]$ and $\Gamma^{\infty}(E)[\lambda]$ by Proposition 1.2.

Note also that $\mathcal{S}^{\bullet}(E)[\lambda]$ is no longer graded with respect to $*_{\text {weyl }}$ but only filtered.

Lemma 6.4. The graded algebra $\mathfrak{G r}^{\bullet}\left(\mathcal{S}^{\bullet}(E)[\lambda], *_{\text {Weyl }}\right)$ is canonically isomorphic to the symmetric algebra $\mathcal{S}^{\bullet}(E)[\lambda]$.

Proof. This is an immediate consequence of Proposition 1.2.

Proposition 6.5. $\left(\mathcal{S}^{\bullet}(E)[\lambda], *_{\text {weyl }}\right)$ is isomorphic to $\mathcal{U}(E)$ via $\phi$.

Proof. It remains to show that $\phi$ is injective. Here we can rely on the complete symbol calculus for Lie algebroids as established in [40, Theorem 3] which is (as our construction) based on the choice of a connection $\nabla$. 


\section{Remark 6.6.}

i) Since $*_{\text {weyl }}$ converges trivially on $\mathcal{S}^{\bullet}(E)[\lambda]$ for any value $\lambda=\hbar \in \mathbb{C}$, we can also pass to the universal enveloping algebra of the Lie algebroid where $\lambda$ is replaced by $\hbar$. This finally establishes the contact to the work of Nistor, Weinstein, and $\mathrm{Xu}$ [40].

ii) For the case $E=\mathfrak{g}$, the above construction reproduces the results of Gutt [22].

iii) For $\kappa \neq \frac{1}{2}$, we also obtain isomorphisms of $\left(\mathcal{S}^{\bullet}(E)[\lambda], *_{\kappa}\right)$ with $\mathcal{U}(E)$ as all $\kappa$-ordered products are equivalent via the explicit equivalence transformation (5.8), which preserves the subspace $\mathcal{S}^{\bullet}(E)[\lambda] \subseteq \mathcal{S}^{\bullet}(E)[[\lambda]]$ thanks to Proposition $\left.5.5 \mathrm{iv}\right)$.

Remark 6.7 (PBW Approach). One may wonder whether a choice of a PBW isomorphism from the symmetric algebra $\mathcal{S}^{\bullet}(E)[\lambda]$ to the graded algebra $\mathfrak{G r}^{\bullet}(\mathcal{U}(E))$ can be used to give an alternative construction of the star product $*_{\text {weyl }}$. This construction was proposed to us by one of the referees. In fact, a posteriori Proposition 6.5 shows that this is possible by using the isomorphism $\phi$ and its inverse described above. However, in this approach one would end up with a deformation quantization of the symmetric algebra $\mathcal{S}^{\bullet}(E)[\lambda]$, even homogeneous, of which it is a priori not clear whether it will extend to a differential star product on $C^{\infty}\left(E^{*}\right)[[\lambda]]$. The point is that the choice of $\phi$ can also be made differently thereby immediately destroying the property that the resulting product is bidifferential. Ultimately, the reason why it works with $\phi$ is a rather involved combinatorial problem: in the Lie algebra case studied in [22] a detailed analysis of the $\mathrm{BCH}$ series was necessary to prove this.

Example 6.8 (Non-differential PBW isomorphism). In [3] an explicit counter example was given in the case of a cotangent bundle $E^{*}=T^{*} \mathbb{R}$ of the real line of a linear isomorphism between $\mathcal{S}\left(E^{*}\right)[\lambda]$ and $\mathcal{U}(E)$ compatible with the filtering but not leading to a bidifferential star product: in this case, the universal enveloping algebra is the algebra of differential operators on $\mathbb{R}$ and a candidate for a linear isomorphism like $\phi^{-1}$ is a quantization map

$$
\varrho: \operatorname{Pol}\left(T^{*} \mathbb{R}\right) \longrightarrow \operatorname{Diffop}(\mathbb{R})
$$

which is completely specified by its values on $\varrho\left(\chi p^{k}\right)$ for $\chi \in C^{\infty}(\mathbb{R})$ and $k \in \mathbb{N}_{0}$ where $p$ denotes the coordinate along the fibers. Then using $\varrho\left(\chi p^{k}\right)=\left(\frac{\lambda}{\mathrm{i}}\right)^{k} \chi \frac{\partial^{k}}{\partial x^{k}}$ for $k \neq 2$ but

$$
\varrho\left(\chi p^{2}\right)=\chi\left(-\lambda^{2} \frac{\partial^{2}}{\partial x^{2}}+\lambda \frac{\partial}{\partial x}\right)
$$

yields an isomorphism such that the pulled back product is not bidifferential (but still homogeneous). In view of this counter example the property of $*_{\text {weyl }}$ to be bidifferential is far from being trivial. In fact, we are not aware of a simple proof of this fact, even for the above isomorphism $\phi$.

In view of this example it remains challenging to show that one can obtain a bidifferential star product for a particular (and geometrically motivated) choice of the PBW isomorphism. One way to prove it independently of our construction is to use the pseudo-differential operator approach of Nistor, Weinstein and Xu: in fact they discuss this construction in [40, Theorem 4] on the level of polynomial functions. However, they do not prove explicitly that the resulting star product is actually bidifferential. But using their integral formulas of the pseudo-differential calculus one should be able to proceed as in the case of the tangent bundle and ordinary pseudodifferential operators. In any case, one should note that their approach is much more analytic than our Fedosov construction: in fact there are several possible generalizations of the Fedosov construction to a completely algebraic framework not referring to underlying geometry at all. 
Remark 6.9. As it was mentioned to us by one of the referees, the $\mathrm{d}_{E}$-closed two-form $B$ can be used to obtain a central extension of the Lie algebroid $E$. For this Lie algebroid, say $\tilde{E}$, one can use the construction for $B=0$ and take an appropriate quotient later to get the deformation quantization of the polynomials $\mathcal{S}^{\bullet}(E)$. However, here it seems to be even more difficult to control whether the result is bidifferential or not. In fact, geometrically the quotient would correspond to a restriction to a submanifold for which it is known that while Poisson structures might be tangential it can happen that no (bidifferential) star product restricts, see the example in [8]. Thus for this case, it seems to be unavoidable to use the above Fedosov machinery to obtain a bidifferential star product.

Again, also in this case one might apply techniques from [40] in so far as the pseudo-differential calculus may be extended to sections of certain line bundles over the base manifold. The resulting star product for the symbols should then incorporate the Chern form of this line bundle as $B$. Of course, this would limit the approach to integral two-forms. For the cotangent bundle case this was shown to work in [2]. We plan to investigate this relation to representation theory of the deformed algebras in some following work including the corresponding Morita theory.

In view of the above remarks one may wonder why one should impose the condition that a star product is differential at all. In fact, there are interesting examples of "star products" defined on polynomial functions on a cotangent bundle which are not differential but enjoy other nice feature, see e.g. the projectively equivariant quantizations in [15]. However, many constructions in deformation quantization rely heavily on the fact that star products are local (and hence locally bidifferential) like e.g. the Cech cohomological approach to the classification $[23,38]$ and to the existence and normalization of traces [24] to mention just a few.

\section{The trace in the unimodular case}

We shall now construct a trace functional for any homogeneous star product on $E^{*}$, in particular for $\star_{\kappa}$ with the choice $B=\lambda B_{1}$ with $B_{1} \in \Gamma^{\infty}\left(\Lambda^{2} E^{*}\right), \mathrm{d}_{E} B_{1}=0$, for the case of a unimodular Lie algebroid. To this end we first have to recall some results from [16] on the modular class, see also [44, 32, 31] as well as [26] for a more algebraic approach. However, we shall use a slightly different presentation.

For a Lie algebroid $E$ there are essentially two ways of defining a divergence of $s \in \Gamma^{\infty}(E)$ : if $\mu \in \Gamma^{\infty}\left(\left|\Lambda^{n}\right| T^{*} M\right)$ is a positive density on $M$ then we can define

$$
\operatorname{div}_{\mu}(s)=\frac{1}{\mu} \mathscr{L}_{\varrho(s)} \mu \in C^{\infty}(M) .
$$

If $\nu \in \Gamma^{\infty}\left(\left|\Lambda^{N}\right| E\right)$ is a positive $E$-density, we analogously can define

$$
\operatorname{div}_{\nu}(s)=\frac{1}{\nu} \mathscr{L}_{s}^{E} \nu \in C^{\infty}(M)
$$

where we use the fact that the $E$-Lie derivative also acts on the $E$-densities in the usual way, see e.g. [43, Section 2.2.5] for an elementary introduction to the calculus of densities. Alternatively, and probably more familiar, one can use volume forms instead of densities, provided $M$ as well as $E$ are orientable. For $u, v \in C^{\infty}(M)$ and $s, t \in \Gamma^{\infty}(E)$ we have the usual identities

$$
\begin{aligned}
& \operatorname{div}_{\mu}(u s)=u \operatorname{div}_{\mu}(s)+\varrho(s) u, \\
& \operatorname{div}_{\mathrm{e}^{v} \mu}(s)=\operatorname{div}_{\mu}(s)+\varrho(s) v, \\
& \operatorname{div}_{\mu}\left([s, t]_{E}\right)=\varrho(s) \operatorname{div}_{\mu}(t)-\varrho(t) \operatorname{div}_{\mu}(s), \\
& \operatorname{div}_{\nu}(u s)=u \operatorname{div}_{\nu}(s)-\varrho(s) u
\end{aligned}
$$




$$
\begin{aligned}
& \operatorname{div}_{\mathrm{e}^{v}}(s)=\operatorname{div}_{\nu}(s)+\varrho(s) v, \\
& \operatorname{div}_{\nu}\left([s, t]_{E}\right)=\varrho(s) \operatorname{div}_{\nu}(t)-\varrho(t) \operatorname{div}_{\nu}(s) .
\end{aligned}
$$

Note the different signs in (7.1) and (7.4): this implies that the map

$$
s \mapsto \operatorname{tr} \operatorname{ad}(s)=\operatorname{div}_{\mu}(s)+\operatorname{div}_{\nu}(s)
$$

is $C^{\infty}(M)$-linear and hence $\operatorname{tr} a d \in \Gamma^{\infty}\left(E^{*}\right)$. From (7.3) and (7.6) we see that $\mathrm{d}_{E} \operatorname{tr}$ ad $=0$ and from (7.2) and (7.5) it follows that the dependence of $\operatorname{tr}$ ad on $\mu$ and $\nu$ is only via $\mathrm{d}_{E}$-exact terms whence $[\operatorname{trad}] \in \mathrm{H}_{E}^{1}(M)$ is a well-defined class [16]:

Definition 7.1 (Modular class). The class $[\operatorname{trad}] \in \mathrm{H}_{E}^{1}(M)$ is called the modular class of $E$ and $E$ is called unimodular if $[\mathrm{tr} \mathrm{ad}]=0$.

In fact, tr ad can be seen as the 'trace of the adjoint representation', even though in general the adjoint representation 'ad' is not really defined in the sense of a Lie algebroid representation. In the unimodular case we can find $\mu$ and $\nu$ such that $\operatorname{tr} \operatorname{ad}(s)=0$ for all $s \in \Gamma^{\infty}(E)$.

In [16] the modular class of a Poisson manifold $(M, \theta)$ was related to the modular class of the corresponding Lie algebroid $T^{*} M$ induced by $\theta$ as in Example $2.1 \mathrm{iii}$ ). Here we shall give yet another interpretation of trad in terms of the Poisson manifold $E^{*}$. To this end we first construct a density on $E^{*}$ out of $\mu$ and $\nu$ in the following way. With respect to local coordinates $\left(q^{1}, \ldots, q^{n}, p_{1}, \ldots, p_{N}\right)$ induced by coordinates $x^{1}, \ldots, x^{n}$ on $M$ and local basis sections $e_{1}, \ldots, e_{N}$ of $E$, we define

$$
(\mu \otimes \nu)\left(\frac{\partial}{\partial q^{1}}, \ldots, \frac{\partial}{\partial q^{n}}, \frac{\partial}{\partial p_{1}}, \ldots, \frac{\partial}{\partial p_{N}}\right)=\pi^{*} \mu\left(\frac{\partial}{\partial x^{1}}, \ldots, \frac{\partial}{\partial x^{n}}\right) \pi^{*} \nu\left(e^{1}, \ldots, e^{N}\right) .
$$

The following lemma is a simple computation using the transformation properties of densities.

Lemma 7.2. $\mu \otimes \nu \in \Gamma^{\infty}\left(\left|\Lambda^{n+N}\right| T^{*} E^{*}\right)$ is a globally well-defined positive density on $E^{*}$.

Lemma 7.3. Let $\Omega \in \Gamma^{\infty}\left(\left|\Lambda^{n+N}\right| T^{*} E^{*}\right)$ be a positive density on $E^{*}$. Then the following statements are equivalent:

i) $\Omega$ is constant along the fibres.

ii) There exist positive densities $\mu \in \Gamma^{\infty}\left(\left|\Lambda^{n}\right| T^{*} M\right)$ and $\nu \in \Gamma^{\infty}\left(\left|\Lambda^{N}\right| E\right)$ such that $\Omega=\mu \otimes \nu$.

iii) $\mathscr{L}_{\xi} \Omega=N \Omega$.

Proof. Assume $i$ ) and choose some positive densities $\tilde{\mu} \in \Gamma^{\infty}\left(\left|\Lambda^{n}\right| T^{*} M\right)$ and $\nu \in \Gamma^{\infty}\left(\left|\Lambda^{N}\right| E\right)$. Then we can write $\Omega=f \tilde{\mu} \otimes \nu$ with $f \in C^{\infty}\left(E^{*}\right)$ and $\Omega$ is constant along the fibres. Thus $f=\pi^{*} u$ with $u \in C^{\infty}(M)$ and $\mu=u \tilde{\mu}$ and $\nu$ will fulfill $i i$ ). If $\left.i i\right)$ holds then $\left.i i i\right)$ is a simple computation. Now assume iii) and choose some $\mu$ and $\nu$ as before. Since clearly $\mathscr{L}_{\xi}(\mu \otimes \nu)=N \mu \otimes \nu$ the function $f=\Omega /(\mu \otimes \nu)$ satisfies $\mathscr{L}_{\xi} f=0$. Hence $f$ and thus $\Omega$ are constant along the fibres.

The next proposition is a simple computation using e.g. the local formulas for the Hamiltonian vector field (2.3):

Proposition 7.4. Let $\mu \in \Gamma^{\infty}\left(\left|\Lambda^{n}\right| T^{*} M\right)$ and $\nu \in \Gamma^{\infty}\left(\left|\Lambda^{N}\right| E\right)$ be positive densities. Then we have for $s \in \Gamma^{\infty}(E)$ and $u \in C^{\infty}(M)$

$$
\begin{aligned}
& \mathscr{L}_{X_{\mathfrak{J}(s)}}(\mu \otimes \nu)=\pi^{*}(\operatorname{tr} \operatorname{ad}(s)) \mu \otimes \nu, \\
& \mathscr{L}_{X_{\pi^{*} u}}(\mu \otimes \nu)=0 .
\end{aligned}
$$


We shall now interpret this proposition in terms of the modular vector field on $E^{*}$. Recall that the vertical lift

$$
\Gamma^{\infty}\left(\Lambda^{k} E^{*}\right) \ni \omega \mapsto \omega^{\text {ver }} \in \Gamma^{\infty}\left(\Lambda^{k} T E^{*}\right)
$$

induces a map in cohomology

$$
\mathrm{H}_{E}^{\bullet}(M) \ni[\omega] \mapsto\left[\omega^{\text {ver }}\right] \in \mathrm{H}_{\theta_{E}}^{\bullet}\left(E^{*}\right)
$$

since we have (with our sign conventions) the relation

$$
\left(\mathrm{d}_{E} \omega\right)^{\mathrm{ver}}=-\mathrm{d}_{\theta_{E}} \omega^{\mathrm{ver}},
$$

see e.g. [21, Theorem 15]. Here $\mathrm{H}_{\theta_{E}}^{\bullet}\left(E^{*}\right)$ denotes the Poisson cohomology and $\mathrm{d}_{\theta_{E}}=\llbracket \theta_{E}, \cdot \rrbracket$. In general, the map (7.9) is far from being surjective, the trivial Lie algebroid provides a simple counter-example. However, it turns out to be injective in general:

Theorem 7.5. The vertical lift induces an injective map (7.9) in cohomology.

Proof. Let $\Phi_{t}\left(\alpha_{q}\right)=\mathrm{e}^{t} \alpha_{q}$ be the flow of the Euler vector field $\xi$ on $E^{*}$. For a $k$-vector field $X \in \Gamma^{\infty}\left(\Lambda^{k} T E^{*}\right)$ on $E^{*}$ we consider $X_{t}=\mathrm{e}^{k t} \Phi_{t}^{*} X \in \Gamma^{\infty}\left(\Lambda^{k} T E^{*}\right)$. A simple argument (say in local coordinates) shows that the limit $t \longrightarrow-\infty$ of $X_{t}$ exists and is a vertical lift. We define

$$
\mathrm{P}(X)=\lim _{t \rightarrow-\infty} X_{t}
$$

For a vertical lift $\omega^{\text {ver }}$ we clearly have $\mathrm{P}\left(\omega^{\text {ver }}\right)=\omega^{\text {ver }}$ and $\mathrm{P}$ is a projection onto the vertical lifts $\Gamma^{\infty}\left(\Lambda^{k} E^{*}\right)^{\text {ver }} \subseteq \Gamma^{\infty}\left(\Lambda^{k} T E^{*}\right)$. Since $\theta_{E}$ is homogeneous of degree -1 , i.e. $\mathrm{e}^{-t} \Phi_{t}^{*} \theta_{E}=\theta_{E}$, and since the Schouten bracket is natural with respect to pull-backs we finally obtain by continuity

$$
\mathrm{P}\left(\mathrm{d}_{\theta_{E}} X\right)=\lim _{t \rightarrow-\infty} \mathrm{e}^{k-1} \Phi_{t}^{*} \llbracket \theta_{E}, X \rrbracket=\llbracket \theta_{E}, \lim _{t \rightarrow-\infty} \mathrm{e}^{k} \Phi_{t}^{*} X \rrbracket=\mathrm{d}_{\theta_{E}} \mathrm{P}(X) .
$$

Thus the projection $\mathrm{P}$ descends to cohomology from which the injectivity of (7.9) follows immediately.

Remark 7.6. In particular, this theorem shows that the scalar Chevalley-Eilenberg cohomology of a Lie algebra $\mathfrak{g}$ is injected into the Poisson cohomology of the linear Poisson structure on the dual $\mathfrak{g}^{*}$ of a Lie algebra $\mathfrak{g}$, see [9, Exercise 72]. Note however, that e.g. for Abelian Lie algebras this map is far from being surjective.

Recall that the modular vector field $\Delta_{\Omega} \in \Gamma^{\infty}\left(T E^{*}\right)$ with respect to some positive density $\Omega \in \Gamma^{\infty}\left(\left|\Lambda^{n+N}\right| T^{*} E^{*}\right)$ is defined by

$$
\Delta_{\Omega}(f) \Omega=\mathscr{L}_{X_{f}} \Omega
$$

and gives a Poisson vector field $\Delta_{\Omega}$ which depends on $\Omega$ only via Hamiltonian vector fields. Thus the modular class $\left[\Delta_{\Omega}\right] \in \mathrm{H}_{\theta_{E}}^{1}\left(E^{*}\right)$ is well defined, see e.g. [16, 44]. Since $\operatorname{Pol}^{0}\left(E^{*}\right)$ and $\operatorname{Pol}^{1}\left(E^{*}\right)$ generate $\operatorname{Pol}^{\bullet}\left(E^{*}\right)$ we can conclude from (7.7) and (7.8)

$$
\Delta_{\mu \otimes \nu}=(\operatorname{trad})^{\mathrm{ver}},
$$

whence $\left[\Delta_{\mu \otimes \nu}\right]$ is precisely the image of the modular class under (7.9). From the injectivity of (7.9) we immediately obtain the following corollary, see also [44, Section 7]:

Corollary 7.7. Let $E$ be a Lie algebroid. Then the following statements are equivalent: 
i) $E$ is unimodular in the sense of Lie algebroids.

ii) $\left(E^{*}, \theta_{E}\right)$ is unimodular in the sense of Poisson manifolds.

iii) There exists a positive density $\Omega \in \Gamma^{\infty}\left(\left|\Lambda^{n+N}\right| T^{*} E^{*}\right)$ on $E^{*}$ which is constant along the fibres and satisfies $\mathscr{L}_{X_{f}} \Omega=0$ for all $f \in C^{\infty}\left(E^{*}\right)$.

Now we assume that $E$ and hence $E^{*}$ are unimodular and we choose an appropriate positive density $\Omega \in \Gamma^{\infty}\left(\left|\Lambda^{n+N}\right| T^{*} E^{*}\right)$ on $E^{*}$ which is constant along the fibres with $\Delta_{\Omega}=0$. Then we can proceed literally as in $[4$, Section 8$]$ and [1] to show that the functional

$$
\operatorname{tr}(f)=\int_{E^{*}} f \Omega
$$

for $f \in C_{0}^{\infty}(M)[[\lambda]]$ defines a $\mathbb{C}[[\lambda]]$-linear trace for any homogeneous star product on $E^{*}$. Thus let $\star=\sum_{r=0}^{\infty} \lambda^{r} C_{r}$ be a homogeneous star product quantizing the Poisson bracket $\{\cdot, \cdot\}_{E}$ of $E^{*}$. The first lemma uses the fact that $\Omega$ is constant along the fibre directions and is shown by partial integration.

Lemma 7.8. Let $D: C^{\infty}\left(E^{*}\right) \longrightarrow C^{\infty}\left(E^{*}\right)$ be a homogeneous differential operator of homogeneity $-r$ with $r \geq 1$, i.e. $\left[\mathscr{L}_{\xi}, D\right]=-r D$. Then $\operatorname{tr}(D(f))=0$ for all $f \in C_{0}^{\infty}\left(E^{*}\right)$.

Lemma 7.9. Let $f \in \operatorname{Pol}^{k}\left(E^{*}\right)$ and $g \in C_{0}^{\infty}\left(E^{*}\right)$. Then

$$
\operatorname{tr}(f \star g)=\operatorname{tr}\left(\sum_{r=0}^{k} \lambda^{r} C_{r}(f, g)\right)
$$

and analogously for $\operatorname{tr}(g \star f)$.

Proof. This follows from the preceding lemma as $C_{r}(f, \cdot)$ and $C_{r}(\cdot, f)$ are homogeneous differential operators of homogeneity $k-r$.

Lemma 7.10. Let $f \in \operatorname{Pol}^{\bullet}\left(E^{*}\right)$ and $g \in C_{0}^{\infty}\left(E^{*}\right)$. Then

$$
\operatorname{tr}(f \star g-g \star f)=0 .
$$

Proof. Let $f$ be homogeneous of degree $k$. For $k=0$ the statement is fulfilled by Lemma 7.9. For $k=1$ it is fulfilled by Lemma 7.9 and the unimodularity condition. For $k>1$ it is sufficient to consider $f=X_{1} \star \cdots \star X_{k}$ with $X_{i}$ homogeneous of degree 0 or 1 according to Proposition $1.2 \mathrm{iv}$ ). From

$$
\left[X_{1} \star \cdots \star X_{k}, g\right]_{\star}=\left[X_{1}, X_{2} \star \cdots \star X_{k} \star g\right]_{\star}+\left[X_{2} \star \cdots \star X_{k}, g \star X_{1}\right]_{\star}
$$

we conclude (7.11) by induction on $k$.

Theorem 7.11. Let $\star$ be a homogeneous star product on $E^{*}$ and let $\mathrm{tr}$ be the Poisson trace as in (7.10). Then $\operatorname{tr}$ is a trace functional with respect to $\star$, too.

Proof. By the usual Stone-Weierstraß argument and the continuity of the integration the theorem follows from the last lemma.

Remark 7.12. Of course there are much more general statements on the traces of star products available like the cyclic formality theorem, see the discussion in [19]. In the case of complex Lie algebroids, one may also consult [11]. Note however, that in the particular situation we are discussing, the proof is elementary. 


\section{Acknowledgements}

We would like to thank Janusz Grabowski, Simone Gutt, Yvette Kosmann-Schwarzbach and Alan Weinstein for valuable discussions and remarks. Moreover, we thank the referees for many interesting suggestions and remarks.

\section{Editorial comments}

For the benefit of the readers we reproduce here verbatim the suggestion of the referee mentioned in Remark 6.9:

"We believe that most of the results presented in the manuscript under consideration can be recovered in a very simpler way. Namely, to any $\mathbb{C}[[\hbar]]$-valued 2 -cocycle $B_{\hbar}$ as above one can associate a central extension $\widetilde{E}_{\hbar}$ of $E[[\hbar]]$ (defined over $\mathbb{C}[[\hbar]]$ ) and can consider its universal enveloping algebra $U\left(\widetilde{E}_{\hbar}\right)$. But we instead consider the $\hbar$-universal enveloping algebra $U_{\hbar}\left(\widetilde{E}_{\hbar}\right)$ (i.e. we put an $\hbar$ in front of commutators in the defining relations).

Finally, take the quotient $A_{\hbar}$ of $U_{\hbar}\left(\widetilde{E}_{\hbar}\right)$ by $c=\hbar$, where $c$ is the generator of the onedimensional extension. One can prove that $A_{\hbar}$ is a topologically free $\mathbb{C}[[\hbar]]$-module (i.e. $A_{\hbar} \cong$ $\Gamma(M, S(E))[[\hbar]])$, and is a quantization of the $B$-twisted Poisson structure on functions polynomial in the fibers on $E^{*}$. Two such quantized algebras are obviously isomorphic if they come from cohomologous cocycles, and the homogeneity property follows easily from this simple construction in the case when $B_{\hbar}=B$.

The proof that this quantization extends to the full algebra of smooth functions on $E^{*}$ follows from the already known case when $B=0$.

Remark. In the case of a Lie algebra this construction is very well-known (it has been used by Drinfel'd to quantize triangular $r$-matrices, and it is quite standard in the theory of quantum integrable systems). In the case of the cotangent bundle these are called twisted differential operators."

\section{References}

[1] Bieliavsky P., Bordemann M., Gutt S., Waldmann S., Traces for star products on the dual of a Lie algebra, Rev. Math. Phys. 15 (2003), 425-445, math.QA/0202126.

[2] Bordemann M., Neumaier N., Pflaum M.J., Waldmann S., On representations of star product algebras over cotangent spaces on Hermitian line bundles, J. Funct. Anal. 199 (2003), 1-47, math.QA/9811055.

[3] Bordemann M., Neumaier N., Waldmann S., Homogeneous Fedosov star products on cotangent bundles. I. Weyl and standard ordering with differential operator representation, Comm. Math. Phys. 198 (1998), 363-396, q-alg/9707030.

[4] Bordemann M., Neumaier N., Waldmann S., Homogeneous Fedosov star products on cotangent bundles. II. GNS representations, the WKB expansion, traces, and applications, J. Geom. Phys. 29 (1999), 199-234, q-alg/9711016.

[5] Bordemann M., Waldmann S., A Fedosov star product of Wick type for Kähler manifolds, Lett. Math. Phys. 41 (1997), 243-253, q-alg/9605012.

[6] Bursztyn H., Radko O., Gauge equivalence of Dirac structures and symplectic groupoids, Ann. Inst. Fourier (Grenoble) $\mathbf{5 3}$ (2003), 309-337, math.SG/0202099.

[7] Cahen M., Gutt S., Regular * representations of Lie algebras, Lett. Math. Phys. 6 (1982), 395-404.

[8] Cahen M., Gutt S., Rawnsley J., On tangential star products for the coadjoint Poisson structure, Comm. Math. Phys. 180 (1996), 99-108.

[9] Cannas da Silva A., Weinstein A., Geometric models for noncommutative algebras, Berkeley Mathematics Lecture Notes, Vol. 10, American Mathematical Society, Providence, RI, Berkeley Center for Pure and Applied Mathematics, Berkeley, CA, 1999. 
[10] Cattaneo A.S., Felder G., Tomassini L., From local to global deformation quantization of Poisson manifolds, Duke Math. J. 115 (2002), 329-352, math.QA/0012228.

[11] Chemla S., A duality property for complex Lie algebroids, Math. Z. 232 (1999), 367-388.

[12] De Wilde M., Lecomte P.B.A., Existence of star-products and of formal deformations of the Poisson Lie algebra of arbitrary symplectic manifolds, Lett. Math. Phys. 7 (1983), 487-496.

[13] De Wilde M., Lecomte P.B.A., Star-products on cotangent bundles, Lett. Math. Phys. 7 (1983), $235-241$.

[14] Dito G., Kontsevich star product on the dual of a Lie algebra, Lett. Math. Phys. 48 (1999), 307-322, math.QA/9905080.

[15] Duval C., El Gradechi A.M., Ovsienko V., Projectively and conformally invariant star-products, Comm. Math. Phys. 244 (2004), 3-27, math.QA/0301052.

[16] Evens S., Lu J.-H., Weinstein A., Transverse measures, the modular class and a cohomology pairing for Lie algebroids, Quart. J. Math. Oxford Ser. (2) 50 (1999), 417-436, dg-ga/9610008.

[17] Fedosov B.V., A simple geometrical construction of deformation quantization, J. Differential Geom. 40 (1994), 213-238.

[18] Fedosov B.V., Deformation quantization and index theory, Mathematical Topics, Vol. 9, Akademie Verlag, Berlin, 1996.

[19] Felder G., Shoikhet B., Deformation quantization with traces, Lett. Math. Phys. 53 (2000), 75-86, math.QA/0002057.

[20] Fernandes R.L., Lie algebroids, holonomy and characteristic classes, Adv. Math. 170 (2002), 119-179, math.DG/0007132.

[21] Grabowski J., Urbańksi P., Tangent and cotangent lifts and graded Lie algebras associated to Lie algebroids, Ann. Global Anal. Geom. 15 (1997), 447-486, dg-ga/9710013.

[22] Gutt S., An explicit *-product on the cotangent bundle of a Lie group, Lett. Math. Phys. 7 (1983), 249-258.

[23] Gutt S., Rawnsley J., Equivalence of star products on a symplectic manifold; an introduction to Deligne's Čech cohomology classes, J. Geom. Phys. 29 (1999), 347-392.

[24] Gutt S., Rawnsley J., Traces for star products on symplectic manifolds, J. Geom. Phys. 42 (2002), 12-18, math.QA/0105089.

[25] Gutt S., Rawnsley J., Natural star products on symplectic manifolds and quantum moment maps, Lett. Math. Phys. 66 (2003), 123-139, math.SG/0304498.

[26] Huebschmann J., Duality for Lie-Rinehart algebras and the modular class, J. Reine Angew. Math. 510 (1999), 103-159, dg-ga/9702008.

[27] Karabegov A.V., On Fedosov's approach to Deformation Quantization with Separation of Variables, in Conférence Moshé Flato 1999 "Quantization, Deformations, and Symmetries" (September 5-8, 1999, Dijon), Editors G. Dito and D. Sternheimer, Math. Phys. Stud., Vol. 22, Kluwer Acad. Publ., Dordrecht, 2000, 167176, math.QA/9903031.

[28] Karabegov A.V., Schlichenmaier M., Almost-Kähler deformation quantization, Lett. Math. Phys. 57 (2001), 135-148, math.QA/0102169.

[29] Kathotia V., Kontsevich's universal formula for deformation quantization and the Campbell-BakerHausdorff formula, Internat. J. Math. 11 (2000), 523-551, math.QA/9811174.

[30] Kontsevich M., Deformation quantization of Poisson manifolds, Lett. Math. Phys. 66 (2003), 157-216, q-alg/9709040.

[31] Kosmann-Schwarzbach Y., Laurent-Gengoux C., Weinstein A., Modular classes of Lie algebroid morphisms, Transform. Groups 13 (2008), 727-755, arXiv:0712.3021.

[32] Kosmann-Schwarzbach Y., Weinstein A., Relative modular classes of Lie algebroids, C. R. Math. Acad. Sci. Paris 341 (2005), 509-514, math.DG/0508515.

[33] Landsman N.P., Mathematical topics between classical and quantum mechanics, Springer Monographs in Mathematics, Springer-Verlag, New York, 1998.

[34] Landsman N.P., Ramazan B., Quantization of Poisson algebras associated to Lie algebroids, in Papers from the AMS-IMS-SIAM Joint Summer Research Conference "Groupoids in Analysis, Geometry, and Physics" (June 20-24, 1999, Boulder), Editors A. Ramsay, and J. Renault, Contemp. Math., Vol. 282, Amer. Math. Soc., Providence, RI, 2001, 159-192, math-ph/0001005. 
[35] Mackenzie K.C.H., General theory of Lie groupoids and Lie algebroids, London Mathematical Society Lecture Note Series, Vol. 213, Cambridge University Press, Cambridge, 2005.

[36] Nest R., Tsygan B., Deformations of symplectic Lie algebroids, deformations of holomorphic symplectic structures, and index theorems, Asian J. Math. 5 (2001), 599-635, math.QA/9906020.

[37] Neumaier N., Klassifikationsergebnisse in der Deformationsquantisierung, PhD thesis, University of Freiburg, 2001, available at http://idefix.physik.uni-freiburg.de/ $\sim$ nine/.

[38] Neumaier N., Local $\nu$-Euler derivations and Deligne's characteristic class of Fedosov star products and star products of special type, Comm. Math. Phys. 230 (2002), 271-288, math.QA/9905176.

[39] Neumaier N., Universality of Fedosov's construction for star products of Wick type on pseudo-Kähler manifolds, Rep. Math. Phys. 52 (2003), 43-80, math.QA/0204031.

[40] Nistor V., Weinstein A., Xu P., Pseudodifferential operators on differential groupoids, Pacific J. Math. 189 (1999), 117-152, funct-an/9702004.

[41] Omori H., Maeda Y., Yoshioka A., Weyl manifolds and deformation quantization, Adv. Math. 85 (1991), $224-255$.

[42] Rinehart G., Differential forms on general commutative algebras, Trans. Amer. Math. Soc. 108 (1963), $195-222$.

[43] Waldmann S., Poisson-Geometrie und Deformationsquantisierung, Eine Einführung, Springer-Verlag, Heidelberg - Berlin - New York, 2007.

[44] Weinstein A., The modular automorphism group of a Poisson manifold, J. Geom. Phys. 23 (1997), 379-394. 